
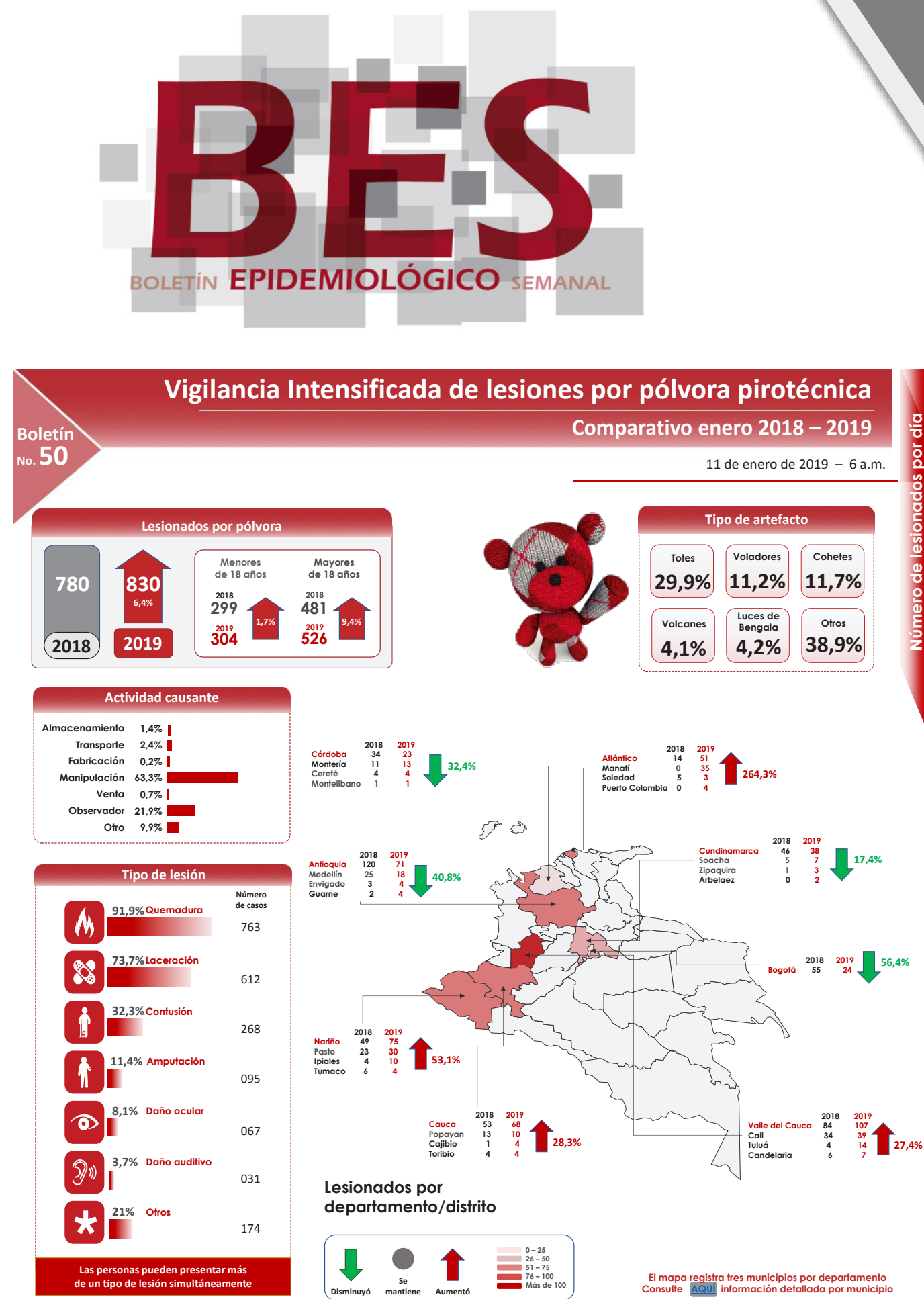

Lesionados por

departamento/distrito

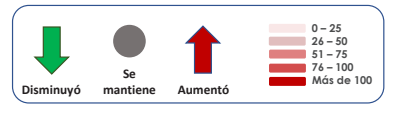

El mapa registra tres municipios por departamento
Consulte
AQUU Información detallada por municipio

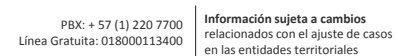

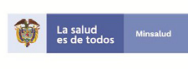

\section{(ㅇ) \\ GOBIERNO \\ DE COLOMBIA}

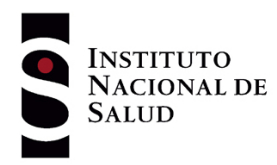

\section{Semana epidemiológica 1}

30 de Diciembre de 2018

a 5 de Enero de 2019

\section{Boletín 50 \\ Vigilancia Intensificada de lesiones por pólvora pirotécnica}

Situación nacional s eventos en salud pública con mayor frecuencia y el cumplimiento de la notificación de las entidades territoriales.
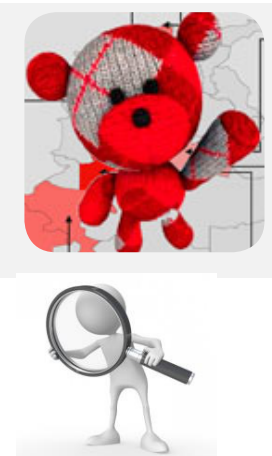

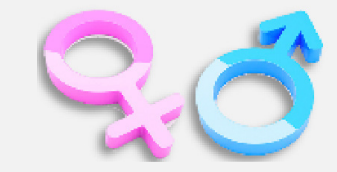

Eventos trazadores Vigilancia en el comportamiento rutinario, siendo un indicador de brotes o emergencias en salud pública.

\section{Mortalidad}

Número de casos notificados al Sivigila, por evento de interés en salud pública

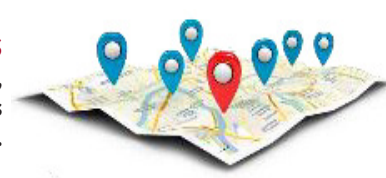

\title{
(3) Sarampión
}

Seguimiento exhaustivo

Brotes y emergencias Actualidad a nivel nacional e internacional

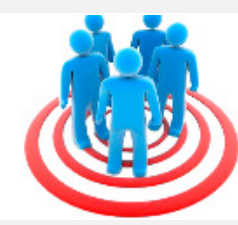

\section{Tablas de mando} Resume la vigilancia rutinaria, permite identificar brotes en salud pública. 


\section{SITUACIÓN NACIONAL}

\section{Tablero de control del análisis de datos de la vigilancia}

En el análisis de esta semana para los eventos que presentan una alta frecuencia (por encima del percentil 50 en el histórico 2012 - 2017), se compara la semana en observación (semana epidemiológica 1) basada en la suma de los casos observados en un periodo epidemiológico (de esta semana y las cuatro semanas previas) y compara el resultado con el promedio de 15 periodos históricos, alrededor de esa semana de evaluación. La ventana histórica de estimación contempla los cinco años previos al año de análisis.

En lo observado para la semana epidemiológica, se identifica que los eventos de mortalidad perinatal y neonatal tardía, Leishmaniasis cutánea, bajo peso al nacer, intoxicaciones y varicela individual; se encuentra por debajo de sus valores esperados. Por otra parte, los demás eventos se encuentran dentro del comportamiento de su notificación histórica. (Gráfica 1).

Gráfica 1

Comparación de los casos notificados priorizados de la semana epidemiológica 1, con su comportamiento histórico para eventos priorizados. Colombia, 2019.

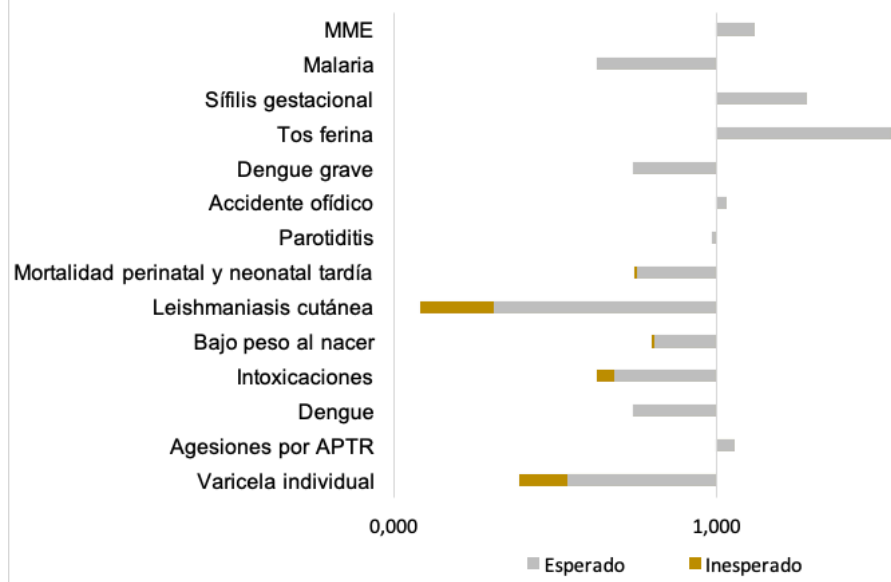

En cuanto a los eventos de baja frecuencia (por debajo del percentil 50 en el histórico 2012 - 2017), se hace uso de la distribución de probabilidades de Poisson, el cual resulta útil para analizar el comportamiento de este tipo de eventos "raros" que -se asume-, ocurren de manera aleatoria en el tiempo. Mediante esta estimación se determina la probabilidad de ocurrencia del evento, según su comportamiento medio anterior. El valor esperado para cada año corresponde al promedio de los últimos cinco años para la misma semana.

En la tabla 1, se observan algunos eventos resaltados que presentan diferencias significativas; en los cuales la probabilidad observada en semana epidemiológica 1, es diferente al comportamiento histórico $(p<0,05)$. En la notificación para esta semana; el evento de Sarampión resaltado en amarillo presenta mayores sus valores observados a los esperados. En los eventos de Chagas, IRAG inusitado, ESAVI y Leishmanisis mucosa, resaltados en gris, sus valores observados no superan a los esperados. Los demás eventos se encuentran dentro del comportamiento histórico establecido. (Tabla 1).

Tabla 1

Comparación de los casos notificados priorizados de la semana epidemiológica 1 con su comportamiento histórico para eventos priorizados. Colombia, 2019

\begin{tabular}{|c|c|c|c|}
\hline Evento & Observado & Esperado & Poisson \\
\hline Chagas & 0 & 28 & 0,0000 \\
\hline Sarampión & 34 & 0 & 0,0000 \\
\hline IRAG inusitado & 27 & 40 & 0,0002 \\
\hline ESAVI & 2 & 8 & 0,0006 \\
\hline Leishmaniasis mucosa & 0 & 10 & 0,0071 \\
\hline Sífilis congénita & 13 & 17 & 0,0628 \\
\hline Leptospirosis & 12 & 30 & 0,0668 \\
\hline Mortalidad materna & 8 & 8 & 0,1040 \\
\hline Fiebre tifoidea y paratifoidea & 0 & 4 & 0,1042 \\
\hline Mortalidad por IRA & 6 & 8 & 0,1167 \\
\hline Mortalidad por EDA 0-4 Años & 2 & 3 & 0,1304 \\
\hline Lepra & 1 & 6 & 0,1697 \\
\hline Tuberculosis fármacorresistente & 0 & 5 & 0,1728 \\
\hline Mortalidad por dengue & 7 & 3 & 0,1929 \\
\hline Rubeola & 3 & 1 & 0,3293 \\
\hline
\end{tabular}

Tema central

Situación $\mathrm{Nal}$

Mortalidad

Trazadores

Sarampión

Brotes

Tablas 


\section{Cumplimiento en la notificación}

La notificación recibida por el Instituto Nacional de Salud correspondiente a la semana epidemiológica 1 de 2019, fue del 100 \% para las unidades notificadoras departamentales y distritales (UND), permaneciendo igual respecto a la misma semana del año 2018 y de la semana anterior. El país cumplió con la meta establecida del $100 \%$ en la notificación semanal.

El reporte de las unidades notificadoras municipales (UNM) a nivel nacional fue de $100 \%$, (1117 de 1117 UNM); aumentó 0,18\% con respecto a la semana anterior y $0,63 \%$ con relación a la misma semana del año 2018. El país cumplió con la meta de $97 \%$ en la notificación semanal, para este nivel de flujo de información (Mapa 1).

El cumplimiento a nivel nacional de las unidades primarias generadoras de datos (UPGD) en esta semana fue de $94,98 \%$ (4923 de 5183 UPGD); disminuyó 0,43\%, respecto a la semana anterior y aumentó 3,4\% a la misma semana epidemiológica del año 2018. Así, el país cumplió con la meta establecida. Sin embargo, el departamento de Guainía; no logró el cumplimiento de la meta para este nivel de flujo de información (Mapa 2).
Mapa 1

Cumplimiento de la notificación por UNM, Colombia, semana epidemiológica 1, 2019

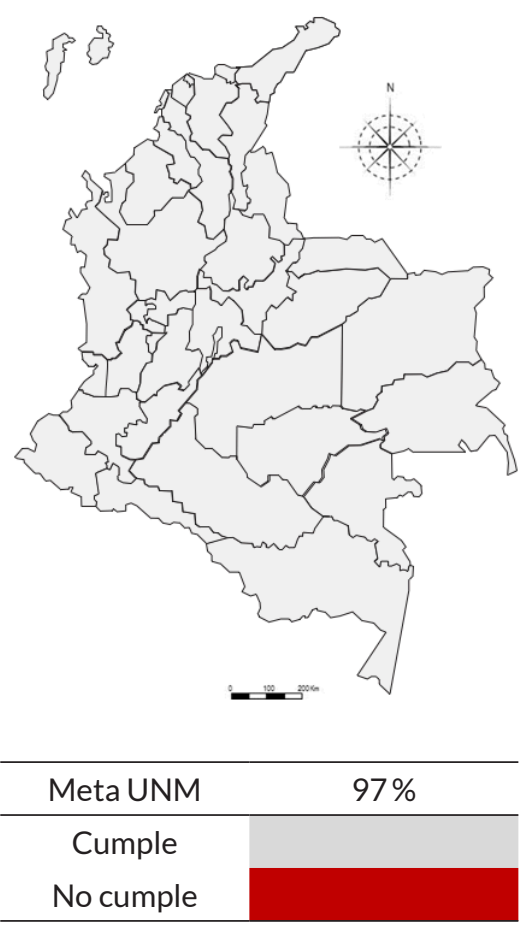

Mapa 2

Cumplimiento de la notificación por UPGD, Colombia, semana epidemiológica 1, 2019

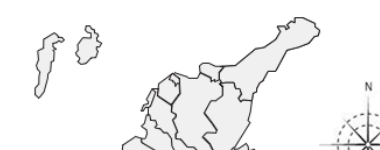
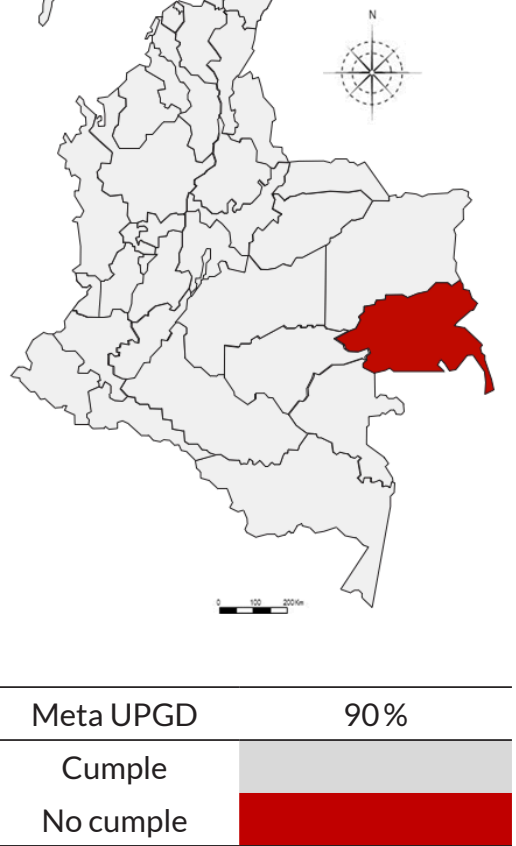

Tema centra

Situación $\mathrm{Na}$

Mortalidad

Trazadores

Sarampión

Brotes

Tablas 


\section{MORTALIDAD}

\section{Mortalidad en menores de 5 años por eventos priorizados}

En la semana epidemiológica 01 de 2019, se notificaron 14 casos probables de mortalidad en menores de 5 años mediante la vigilancia integrada de IRA, EDA y DNT, de estos, ocho corresponden a muertes probables por IRA, tres a DNT y dos a EDA.

\section{Mortalidad por infección respiratoria aguda en menores de 5 años}

En la semana epidemiológica 01 de 2019, se notificaron al Sivigila ocho casos de muerte probable por infección respiratoria aguda, de los cuales seis casos corresponden a esta semana y dos casos se notificaron tardíamente, correspondientes al 2018; en la misma semana de 2018 se notificaron nueve casos.

Respecto al acumulado de muertes notificadas a semana epidemiológica 01 por entidad territorial se observa una disminución en el número de casos en comparación al promedio de casos notificados para el mismo periodo entre los años 2014 a 2018 en Antioquia; En las restantes entidades territoriales no se observan variaciones significativas, este mismo comportamiento se observa en el acumulado de casos notificados para el país.

\section{Mortalidad por enfermedad diarreica aguda}

En la semana epidemiológica 01 de 2019 ingresaron al sistema de vigilancia dos casos probables de mortalidad por enfermedad diarreica aguda en menores de 5 años. En la misma semana de 2018 se notificó un caso.

A semana epidemiológica 01 , Choco presenta una reducción estadísticamente significativa en la notificación de muertes por enfermedad diarreica aguda en menores de cinco años en comparación con el promedio de notificación de los años 2013 a 2018; mientras que en las restantes entidades territoriales no se observan variaciones significativas, se observa el mismo comportamiento en el acumulado de casos notificados para el país.

\section{Mortalidad por y asociada a desnutrición}

En la semana epidemiológica 01 de 2019, se notifican al Sivigila cuatro casos de muertes probables por y asociadas a desnutrición en menores de cinco años, de los cuales tres corresponden a esta semana y un caso fue notificado tardíamente, correspondiente al 2018. En la misma semana de 2018 se notificó un caso. Los casos corresponden a muertes por diferentes patologías que registran desnutrición como uno de los diagnósticos.

A semana epidemiológica 01 ninguna entidad territorial presenta variaciones estadísticamente significativas en comparación con el promedio de casos notificados para el mismo periodo entre los años 2014 - 2018.
Tema central

Situación $\mathrm{Nal}$

Mortalidad

Trazadores

Sarampión

Brotes

Tablas 


\section{Mortalidad materna}

En la semana epidemiológica 01 de 2019, se notificaron al Sivigila ocho casos de muerte materna, correspondientes a esta semana. De los casos notificados seis corresponden a mortalidad materna temprana (ocurridas duranteel embarazo, partoy hasta los 42 días de terminada la gestación), un caso corresponde a mortalidad materna tardía (desde el día 43 hasta un año de terminada la gestación) y un caso corresponde a mortalidad materna por causas coincidente. En la misma semana de 2018 se notificaron cuatro casos de muerte materna temprana. A la fecha, han ingresado al Sivigila ocho casos confirmados de muerte materna, seis corresponden a muertes maternas tempranas, uno corresponde a muerte materna tardía y un caso corresponde a muerte materna producida por causas coincidentes (Tabla2).

Tabla2

Notificación de mortalidad materna, Colombia, semanas epidemiológicas 01, Años 2017-2019.

\begin{tabular}{ccccc} 
Año & Temprana & Tardía & Coincidente & Total \\
2017 & 4 & 1 & 0 & 5 \\
\hline 2018 & 4 & 4 & 2 & 10 \\
\hline 2019 & 6 & 1 & 1 & 8 \\
\hline
\end{tabular}

Fuente: Sivigila, Instituto Nacional de Salud, Colombia, 2016-2018

Al ser la mortalidad materna un evento de baja frecuencia, para su análisis se hace uso de la distribución de probabilidades de Poisson, por medio de la estimación de la probabilidad de ocurrencia del evento según su comportamiento medio entre 2013 y 2018. Para la semana epidemiológica 01 de 2019 se observa un aumento significativo en los casos de muertes maternas tempranas frente al promedio esperado en las entidades territoriales de Nariño, Sucre, Tolima, Valle y Vaupés $(p<0,05)$.
Tabla 3

Entidades territoriales con variaciones respecto al promedio de casos notificados 2013-2018, Colombia, semanas epidemiológicas 01, 2019

\begin{tabular}{|cccc|}
\hline Entidad territorial & Observado & Esperado & Poisson \\
Nariño & 1 & 0 & 0,000 \\
\hline Sucre & 1 & 0 & 0,000 \\
\hline Tolima & 1 & 0 & 0,000 \\
\hline Valle & 1 & 0 & 0,000 \\
\hline Vaupés & 1 & 0 & 0,000 \\
\hline
\end{tabular}

Fuente: Sivigila, Instituto Nacional de Salud, Colombia, 2012-2018

Se notificaron 6 casos de muerte materna temprana residentes en 6 entidades territoriales, encontrando que a semana epidemiológica 01 de 2019 la razón de mortalidad materna nacional preliminar es de 11,0 casos por 100.000 nacidos vivos. La entidad territorial con la razón de mortalidad materna preliminar más alta, mayor a 100 casos por 100.000 nacidos vivos, es Vaupés con 3448,3 , casos por 100.000 nacidos vivos (Gráfica 2).

Gráfica 2

Razón de mortalidad materna por entidad territorial de residencia, Colombia, semanas epidemiológicas 01, 2019

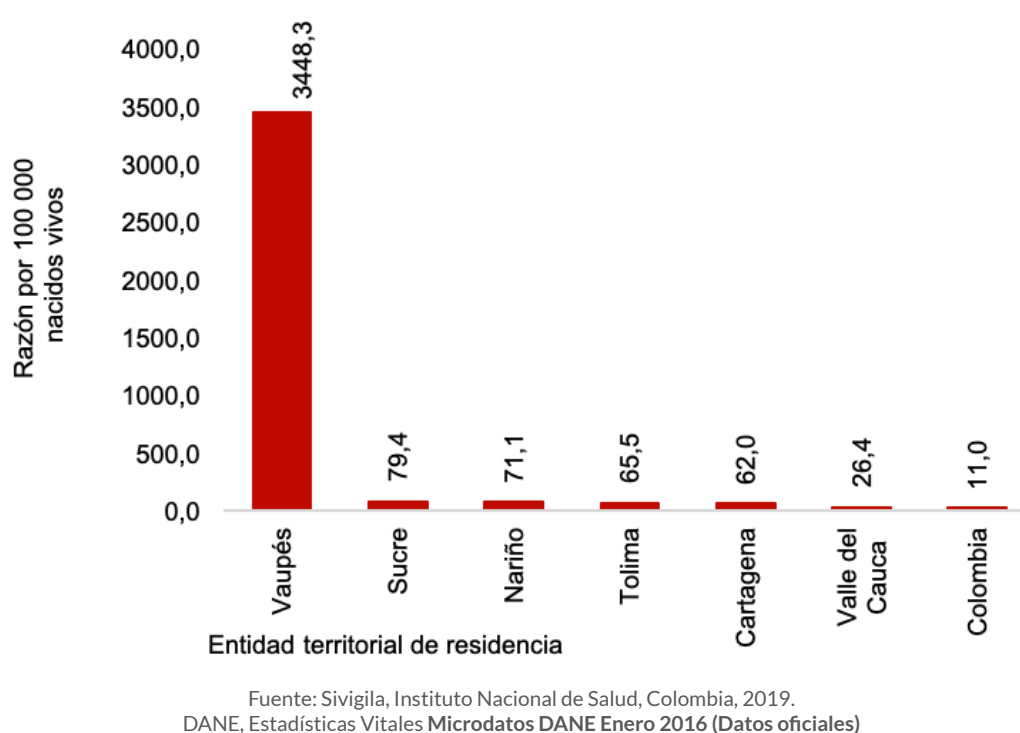

Tema centra

Situación $\mathrm{Nal}$.

Mortalidad

Trazadores

Sarampión

Brotes

Tablas instituto NACIO 


\section{Mortalidad Perinatal y Neonatal}

En la semana epidemiológica 01 de 2019 se notificaron 81 muertes perinatales y neonatales tardías de esta semana. A la fecha, se han notificado al Sivigila 81 casos.

En el análisis para esta semana se comparó lo observado en la semana epidemiológica 01, basándose en la suma de los casos observados en un periodo epidemiológico (de esta semana y las 3 semanas previas); y compara el resultado con el promedio de 15 periodos históricos alrededor de esa semana de evaluación, la ventana histórica de estimación contempla los 5 años previos al año de análisis.

En lo observado para la semana 01 se presenta un aumento para los casos residentes en el Exterior, Vichada, Caquetá y Arauca tienen un incremento en la notificación con respecto a su comportamiento para el mismo periodo de tiempo (gráfica 3).

\section{Gráfica 3}

Notificación de mortalidad perinatal y neonatal tardía por semana epidemiológica, Colombia, semanas epidemiológicas 50-01, 2014-2019

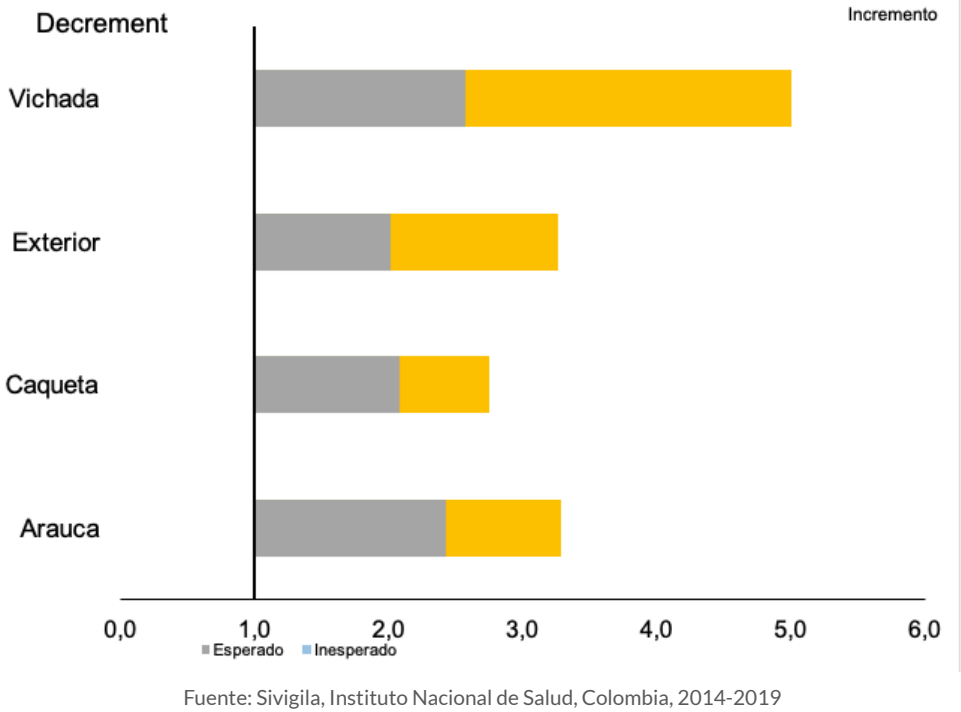

Según el momento de ocurrencia, 31 casos (el 38,3\%) corresponden a muertes perinatales anteparto, 10 casos (el 12,3\%) a muertes perinatales intraparto, 23 casos (el $28,4 \%$ ) a muertes neonatales tempranas, 17 casos (el 21,0\%) a muertes neonatales tardías.

La tasa de mortalidad perinatal y neonatal tardía nacional es de 1,5 muertes por 1.000 nacidos vivos. Quince (15) entidades territoriales superan la tasa nacional. Vaupés con 33,3, Putumayo 5,1, Caquetá con 4,9, Norte de Santander con 4,8, La Guajira con 4,4, Quindío con 4,0, Nariño y Atlántico con 2,8 muertes por 1.000 nacidos vivos, registran las tasas más altas a nivel nacional (gráfica 4).

\section{Gráfica 4}

Tasa de mortalidad perinatal y neonatal tardía por entidad territorial de residencia, Colombia, semana epidemiológica 01, 2019

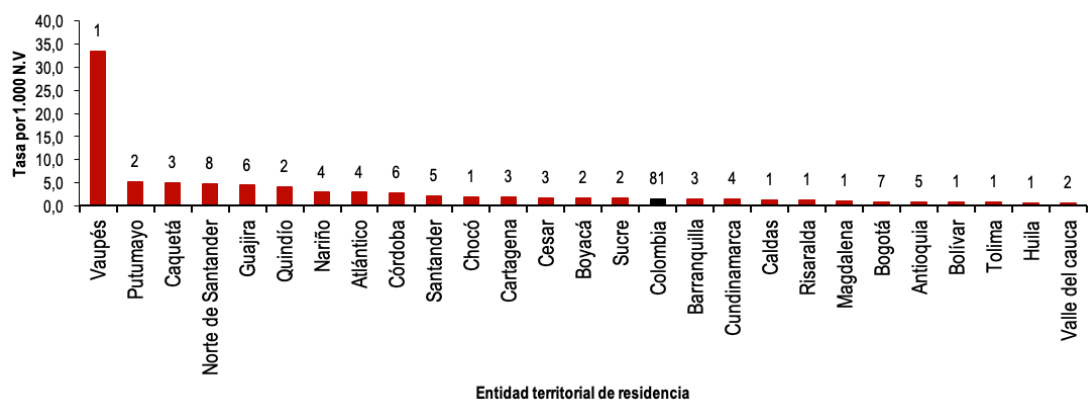

Fuente: Sivigila, Instituto Nacional de Salud, Colombia, 2018. DANE, Estadísticas Vitales, Cifras oficiales nacimientos enero 2016
Tema central

Situación Nal.

Mortalidad

Trazadores

Sarampión

Brotes

Tablas 


\section{EVENTOS TRAZADORES}

\section{Infección respiratoria aguda}

\section{Vigilancia de infección respiratoria aguda grave inusitada}

En la semana epidemiológica 01 de 2019, se notificaron al Sivigila 25 casos de infección respiratoria aguda grave inusitada, 10 de esta semana y 14 de semanas anteriores correspondientes al 2018; no se han identificado virus nuevos o de alta patogenicidad en los casos de infección respiratoria aguda grave inusitada notificados.

Para la semana epidemiológica 01 de 2019 comportamiento de la notificación de casos de IRAG inusitado se encuentra por debajo del promedio histórico de notificación de las semanas 01 a 52 de 2018 (gráfica 5).

\section{Gráfica 5}

Comparación de la notificación de Infección respiratoria aguda grave inusitada, Colombia, semanas epidemiológicas 01, 2019

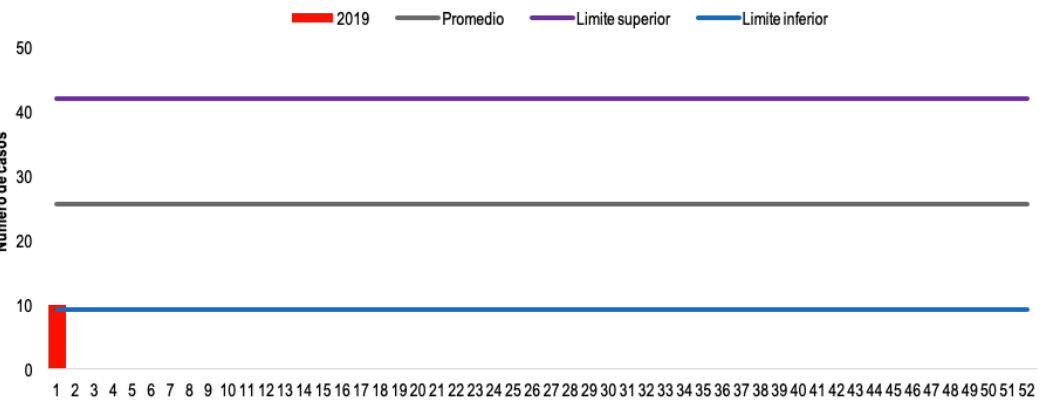
Semana eppidemiologica

\section{Vigilancia centinela de enfermedad similar a la influenza e infección respiratoria aguda grave}

En la semana epidemiológica 01 de 2019, se notificaron al Sivigila 81 casos mediante la estrategia de vigilancia centinela de enfermedad similar a la influenza e infección respiratoria aguda grave, 23 de esta semana y 50 de semanas anteriores correspondientes al 2018; de los 81 casos notificados el $100 \%$ corresponden a pacientes con infección respiratoria aguda grave, durante esta semana no se notificaron pacientes con enfermedad similar a la influenza.

Para la semana epidemiológica 01 la notificación de casos de ESIIRAG centinela se encuentra por debajo del límite inferior histórico y del umbral estacional (gráfica 6).

\section{Gráfica 6}

Comportamiento de la notificación de casos de enfermedad similar a la influenza e infección respiratoria aguda grave, Colombia, semana epidemiológica 1, 2012-2019

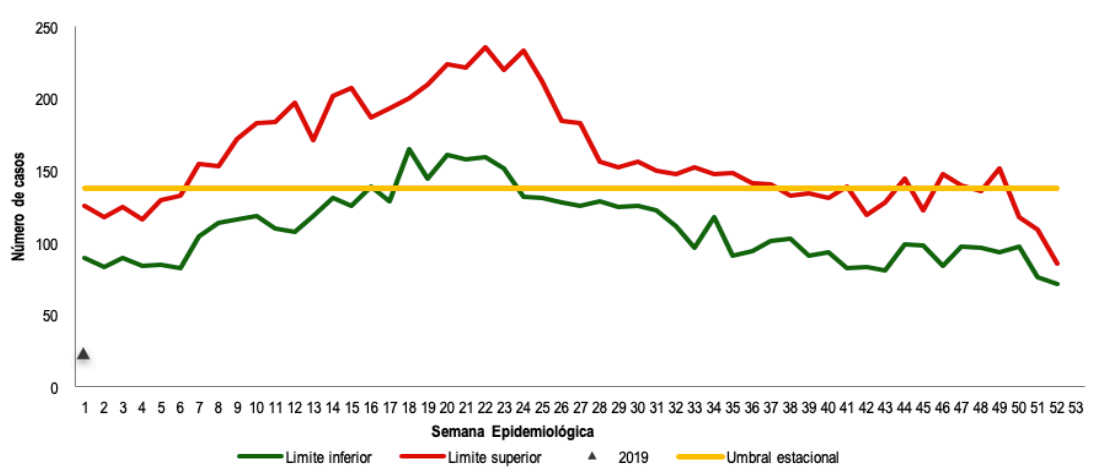

Antioquia, Bogotá, Guaviare, Nariño, Meta, Norte de Santander, Tolima y Valle del Cauca notificaron casos durante la semana epidemiológica 01; las UPGD centinelas que aparecen sin casos no cumplieron el indicador de notificación para esta semana (tabla 4). 


\section{Tabla 4}

Casos notificados de enfermedad similar a la influenza e infección respiratoria aguda grave por entidad territorial y UPGD centinela, Colombia, semana epidemiológica 1, 2019

\begin{tabular}{ccc} 
Entidad Territorial & UPGD Centinela & Casos \\
Antioquia & Fundación Hospitalaria San Vicente de Paul & 12 \\
\hline Barranquilla & Clínica General del Norte & 0 \\
\hline \multirow{2}{*}{ Bogotá } & Hospital el Tunal & 2 \\
\cline { 2 - 3 } Cartagena & Fundación Cardioinfantil & 16 \\
\hline Guaviare & Hospital Infantil Napoleón Franco Pareja & 0 \\
\hline Meta & Laboratorio Departamental de Salud Publica & 1 \\
\hline \multirow{2}{*}{ Nariño } & Fundación Hospital San Pedro & 2 \\
\hline Norte de Santander & Hospital Infantil Los Ángeles & 4 \\
\hline Tolima & Hospital Universitario Erasmo Meoz & 13 \\
\hline Valle del Cauca & Hospital Federico Lleras Acosta & 4 \\
\hline & Fundación Valle del Lili & 20 \\
\hline
\end{tabular}

\section{Vigilancia de morbilidad por infección respiratoria aguda}

En el análisis de la morbilidad por infección respiratoria aguda, mediante el método para comparar el comportamiento observado (semana epidemiológica01y las tres semanas previas) con la mediana histórica alrededor de la semana de evaluación (años 2014 a 2018), la razón observada de casos de infección respiratoria aguda presenta aumento en las consultas externas, urgencias, hospitalización en sala general y $\mathrm{UCI}$ sin diferencias estadísticamente significativas en la morbilidad por IRA.

En esta semana se notificaron 94630 consultas externas y urgencias por infección respiratoria aguda (IRA) de 1261274 consultas externas y urgencias por todas las causas, obteniendo una proporción del 7,5\%.

En las consultas externas y urgencias por IRA, los valores observados se encuentran por debajo de la razón esperada y límite superior histórico (gráfica 7).

\section{Gráfica 7}

Comparación de consultas externas y urgencias por infección respiratoria aguda a semana epidemiológica 1 de 2019 con su comportamiento histórico,

Colombia, 2014-2018

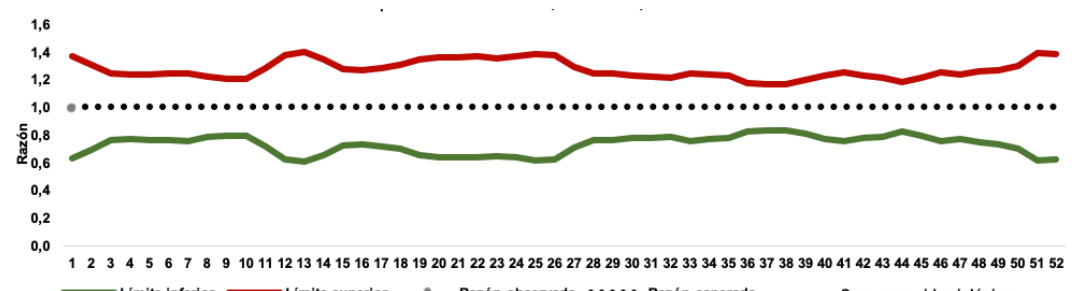

Durante la semana se notificaron 2980 hospitalizaciones por infección respiratoria aguda grave (IRAG) de 41815 hospitalizaciones por todas las causas, obteniendo una proporción del 7,1\%.

En la semana se notificaron 325 hospitalizaciones en UCI por IRAG de 4021 hospitalizaciones por todas las causas, obteniendo una proporción del 8,1\%.

En las hospitalizaciones por IRAG, los valores observados se encuentran por debajo de la razón esperada y límite superior histórico (gráfica 8).

\section{Gráfica 8}

Comparación de hospitalizaciones por infección respiratoria aguda grave a semana epidemiológica 1 de 2019 con su comportamiento histórico, Colombia, 2014-2018

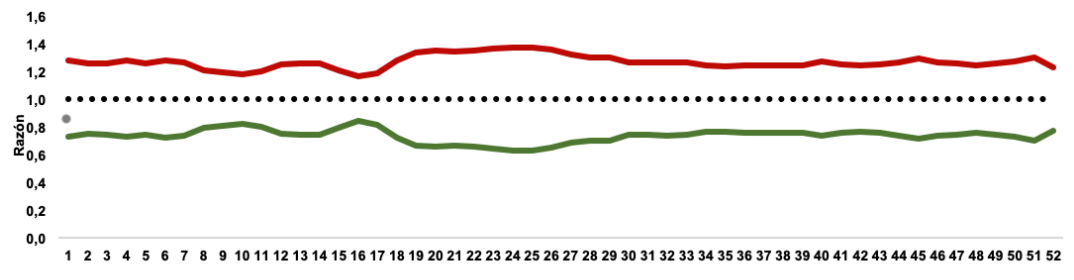
—Limite inferior —Limite superior - Razón observada $\cdots . .$. Razón esperada Semanas epidemiológicas
Tema central

Situación $\mathrm{Nal}$

Mortalidad

Trazadores

Sarampión

Brotes

Tablas 


\section{Chikungunya}

En la semana epidemiológica 01 de 2019, se notificó al Sivigila un caso de chikungunya, observándose una reducción del $94.7 \%$ con respecto a la notificación de la misma semana del año 2018, en el cual se notificaron 19 casos. A la fecha, el caso ingresado fue clasificado como confirmados por clínica (gráfica 9).

\section{Gráfica 9}

Casos notificados de chikungunya, Colombia, semanas epidemiológicas 01, 2018-2019

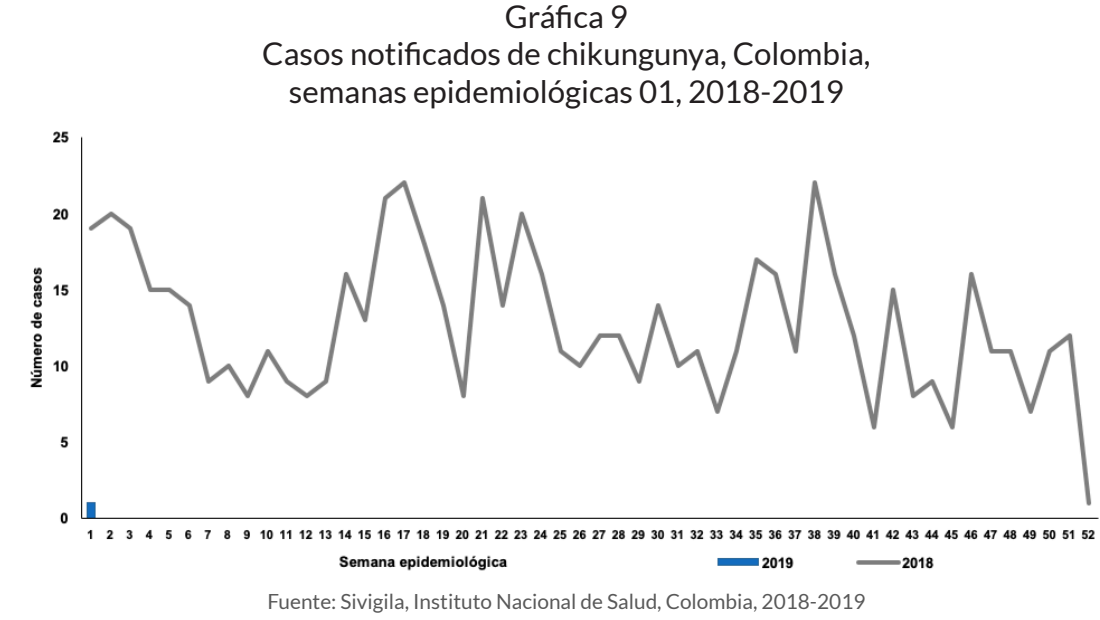

Por procedencia, durante la semana epidemiológica 01 de 2019, Cesar notificó el 100,0\% de los casos (tabla 5).
Tabla 5

Casos notificados de chikungunya por entidad territorial de procedencia, Colombia, semanas epidemiológicas 1, 2019

\begin{tabular}{|c|c|c|c|c|}
\hline $\begin{array}{l}\text { Entidad territorial } \\
\text { de procedencia }\end{array}$ & $\begin{array}{l}\text { Casos confirmados } \\
\text { por clínica }\end{array}$ & $\begin{array}{c}\text { Casos Confirmados } \\
\text { por laboratorio }\end{array}$ & $\begin{array}{c}\text { Casos } \\
\text { sospechosos }\end{array}$ & Total \\
\hline Amazonas & 0 & 0 & 0 & 0 \\
\hline Antioquia & 0 & 0 & 0 & 0 \\
\hline Arauca & 0 & 0 & 0 & 0 \\
\hline Atlántico & 0 & 0 & 0 & 0 \\
\hline Barranquilla & 0 & 0 & 0 & 0 \\
\hline Bogotá & 0 & 0 & 0 & 0 \\
\hline Bolívar & 0 & 0 & 0 & 0 \\
\hline Boyacá & 0 & 0 & 0 & 0 \\
\hline Buenaventura & 0 & 0 & 0 & 0 \\
\hline Caldas & 0 & 0 & 0 & 0 \\
\hline Caquetá & 0 & 0 & 0 & 0 \\
\hline Cartagena & 0 & 0 & 0 & 0 \\
\hline Casanare & 0 & 0 & 0 & 0 \\
\hline Cauca & 0 & 0 & 0 & 0 \\
\hline Cesar & 1 & 0 & 0 & 1 \\
\hline Chocó & 0 & 0 & 0 & 0 \\
\hline Córdoba & 0 & 0 & 0 & 0 \\
\hline Cundinamarca & 0 & 0 & 0 & 0 \\
\hline Guainía & 0 & 0 & 0 & 0 \\
\hline Guajira & 0 & 0 & 0 & 0 \\
\hline Guaviare & 0 & 0 & 0 & 0 \\
\hline Huila & 0 & 0 & 0 & 0 \\
\hline Magdalena & 0 & 0 & 0 & 0 \\
\hline Meta & 0 & 0 & 0 & 0 \\
\hline Nariño & 0 & 0 & 0 & 0 \\
\hline Norte Santander & 0 & 0 & 0 & 0 \\
\hline Putumayo & 0 & 0 & 0 & 0 \\
\hline Quindío & 0 & 0 & 0 & 0 \\
\hline Risaralda & 0 & 0 & 0 & 0 \\
\hline San Andrés & 0 & 0 & 0 & 0 \\
\hline Santander & 0 & 0 & 0 & 0 \\
\hline Santa Marta D.E. & 0 & 0 & 0 & 0 \\
\hline Sucre & 0 & 0 & 0 & 0 \\
\hline Tolima & 0 & 0 & 0 & 0 \\
\hline Valle del Cauca & 0 & 0 & 0 & 0 \\
\hline Total & 1 & 0 & 0 & 1 \\
\hline
\end{tabular}

Fuente: Sivigila, Instituto Nacional de Salud, Colombia, 2019 
El caso notificado corresponde a un paciente femenino de 24 años de edad, afiliada al régimen contributivo, de pertenencia étnica otros y procedente del área urbana.

La incidencia nacional de chikungunya es de 0.004 casos por 100000 habitantes de la población a riesgo y la entidad territorial de Cesar presentó una incidencia de 0.12 casos por 100.000 habitantes de la población a riesgo. De acuerdo con el análisis del comportamiento del evento por entidad territorial, mediante la distribución de probabilidades de Poisson para la semana epidemiológica 01 de 2019, se observa que la entidad territorial de Valle del Cauca presentó un decremento significativo frente a lo esperado $(p<0,05)$ y ninguna entidad territorial presentó un incremento significativo frente a lo esperado $(p<0,05)$.

En la semana epidemiológica 01 del año 2019 se evidencia un descenso general de casos comparado con la notificación del año 2018, con una reducción del $94.7 \%$. Durante la semana epidemiológica 01 de 2019 el evento se ubicó por debajo del límite inferior (Gráfica 10).

\section{Gráfica 10}

Comportamiento epidemiológico de chikungunya, Colombia, año 2017 a semana epidemiológica 01 de 2019

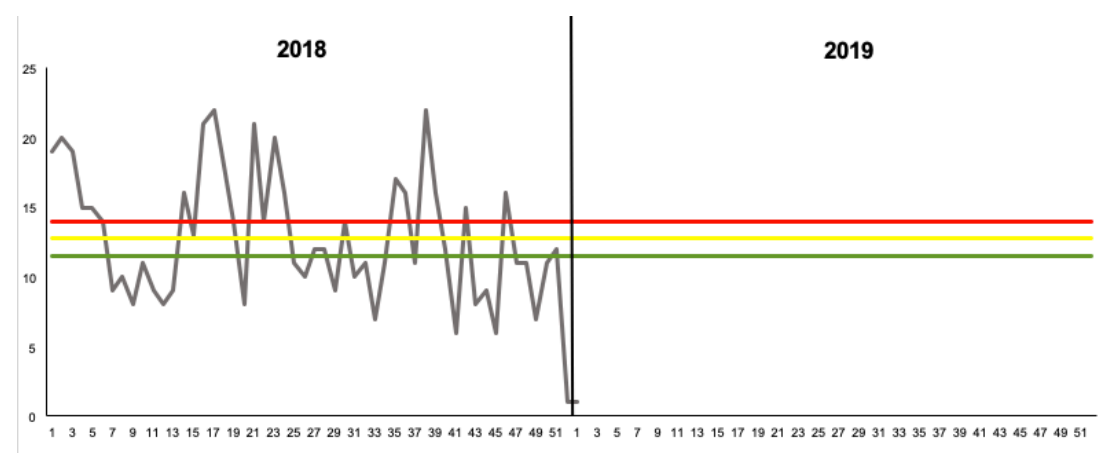
—Casos - Promedio —um Sup —umb

Fuente: Sivigila, Instituto Nacional de Salud, Colombia, 2018 - 2019

\section{Dengue}

En la semana epidemiológica 01 de 2019 se notificaron al Sivigila 605 casos probables de dengue; 262 casos (el 43,3\%) sin signos de alarma; 329 casos (el $54,4 \%$ ) con signos de alarma y 14 casos (el $2,3 \%)$ de dengue grave.

Por procedencia Norte de Santander, Magdalena, Tolima, Cesar, Antioquia, Meta, Huila, Córdoba y Putumayo notificaron el 62,0 \% de los casos (tabla 6).

\section{Tabla 6}

Casos notificados de dengue por entidad territorial de procedencia y clasificación, Colombia, semanas epidemiológicas 01, 2019

\begin{tabular}{cccccc} 
Entidad territorial & $\begin{array}{c}\text { Casos de } \\
\text { dengue }\end{array}$ & $\begin{array}{c}\text { Frecuencia } \\
\text { relativa }\end{array}$ & $\begin{array}{c}\text { Casos de } \\
\text { dengue grave }\end{array}$ & $\begin{array}{c}\text { Frecuencia } \\
\text { relativa }\end{array}$ & $\begin{array}{c}\text { Total } \\
\text { dengue }\end{array}$ \\
\hline Magdalander & 64 & 10,8 & 0 & 0,0 & 64 \\
\hline Tolima & 53 & 9,0 & 0 & 0,0 & 53 \\
\hline Cesar & 52 & 8,8 & 0 & 0,0 & 52 \\
\hline Antioquia & 45 & 7,6 & 2 & 14,3 & 47 \\
\hline Meta & 39 & 6,6 & 0 & 0,0 & 39 \\
\hline Huila & 35 & 5,9 & 1 & 7,1 & 36 \\
\hline Córdoba & 33 & 5,6 & 0 & 0,0 & 33 \\
\hline Putumayo & 30 & 5,1 & 0 & 0,0 & 30 \\
\hline Santander & 24 & 4,1 & 1 & 7,1 & 25 \\
\hline Valle del Cauca & 22 & 3,7 & 1 & 7,1 & 23 \\
\hline Cartagena & 21 & 3,6 & 1 & 7,1 & 22 \\
\hline Sucre & 22 & 3,7 & 0 & 0,0 & 22 \\
\hline Cundinamarca & 20 & 3,4 & 1 & 7,1 & 21 \\
\hline Bolívar & 18 & 3,0 & 2 & 14,3 & 20 \\
\hline Atlántico & 18 & 3,0 & 2 & 14,3 & 20 \\
\hline Barranquilla & 16 & 2,7 & 1 & 7,1 & 17 \\
\hline Arauca & 12 & 2,0 & 1 & 7,1 & 13 \\
\hline La Guajira & 11 & 1,9 & 0 & 0 & 11 \\
\hline Quindío & 9 & 1,5 & 0 & 0 & 9 \\
\hline Casanare & 9 & 1,5 & 0 & 0 & 9 \\
\hline Santa Marta & 7 & 1,2 & 0 & 0 & 7 \\
\hline Risaralda & 7 & 1,2 & 0 & 0 & 7 \\
\hline Boyacá & 6 & 1,0 & 0 & 0 & 6 \\
\hline Guaviare & 5 & 0,8 & 0 & 0 & 5 \\
\hline Cauca & 4 & 0,7 & 0 & 0 & 4 \\
\hline Caldas & 3 & 0,5 & 1 & 7,1 & 4 \\
\hline Nariño & 3 & 0,5 & 0 & 0 & 3 \\
\hline Exterior & 2 & 0,3 & 0 & 0 & 2 \\
\hline & 1 & 0,2 & 0 & 0 & 1 \\
\hline & & & & 0 & \\
\hline
\end{tabular}

Fuente: Sivigila, Instituto Nacional de Salud, Colombia, 2019
Tema central

Situación Nal.

Mortalidad

Trazadores

Sarampión

Brotes

Tablas 
Reportaron casos procedentes de 26 entidades territoriales, tres distritos y 184 municipios. A nivel municipal, la mayor proporción de casos de dengue se presentan en Cúcuta con el 6,3\%, Cartagena con el 3,7\%, Espinal con el 3,4\%, Aracataca con el 3,0\%, Villavicencio y Neiva con el 2,9\% cada uno; Valledupar con el 2,4\%, Montería con el 2,2 \%, Barranquilla con el 2,0\%, Los Patios con el 1,9\%, Cali y Orito con el 1,7\% cada uno; Medellín y Fundación con el 1,5 \% cada uno; que concentran el 37,1\% de la notificación. Para dengue grave se notificaron casos procedentes de los municipios de Aguachica (dos casos), Floridablanca, Villavicencio, Cali, Santa Rosa, Galapa, Puerto Salgar, Caloto, Puerto Caicedo, Santa Rosa del Sur, Barranquilla, Palmito y Girardot, cada uno con un caso.

El 52,2 \% de los casos de dengue se registró en el sexo masculino; el $56,7 \%$ de los casos pertenecen al régimen subsidiado y un $4,6 \%$ refieren no afiliación; el 19,1\% de los casos de dengue y el 21,4\% de los casos notificados de dengue grave se registraron en menores de 5 años. Se han notificado 14 casos (el 2,3\%) en población indígena, 12 casos (el 2,0 \%) en afrocolombianos y gestantes en dos casos (el $0,7 \%)$

Del total de casos de dengue con signos de alarma fueron hospitalizados el 73,3\% (gráfica 11).

\section{Gráfica 11}

Hospitalizados de casos notificados de dengue con signos de alarma por entidad notificadora, Colombia, semanas epidemiológicas 01, 2019

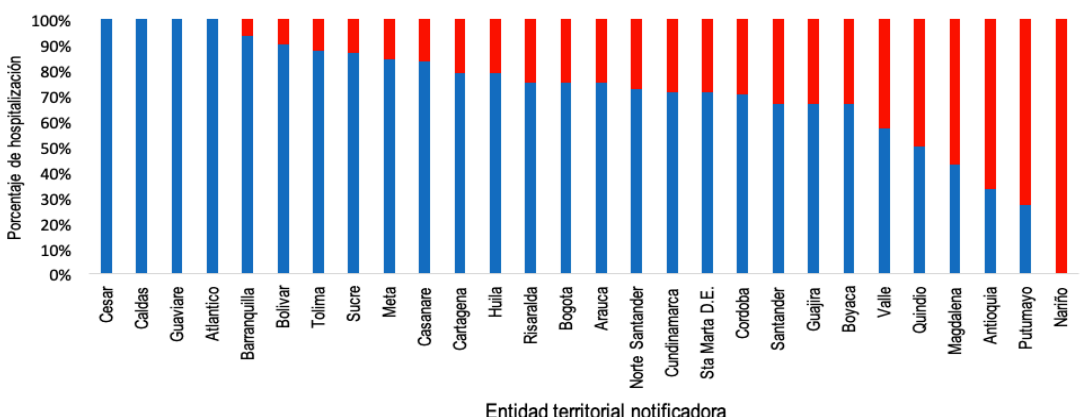

Fuente: Sivigila, Instituto Nacional de Salud, Colombia, 2019
Para dengue grave la hospitalización fue del $85,7 \%$ de los casos (12/14). Con indicadores de hospitalización por debajo del $100 \%$ para los departamentos de Cundinamarca con el $50 \%$ (1/2 casos) y Valle del Cauca el 50 \% (1/2 casos) (gráfica 12).

Gráfica 12

Hospitalizados de casos notificados de dengue grave por entidad notificadora, Colombia, semanas epidemiológicas 01, 2019

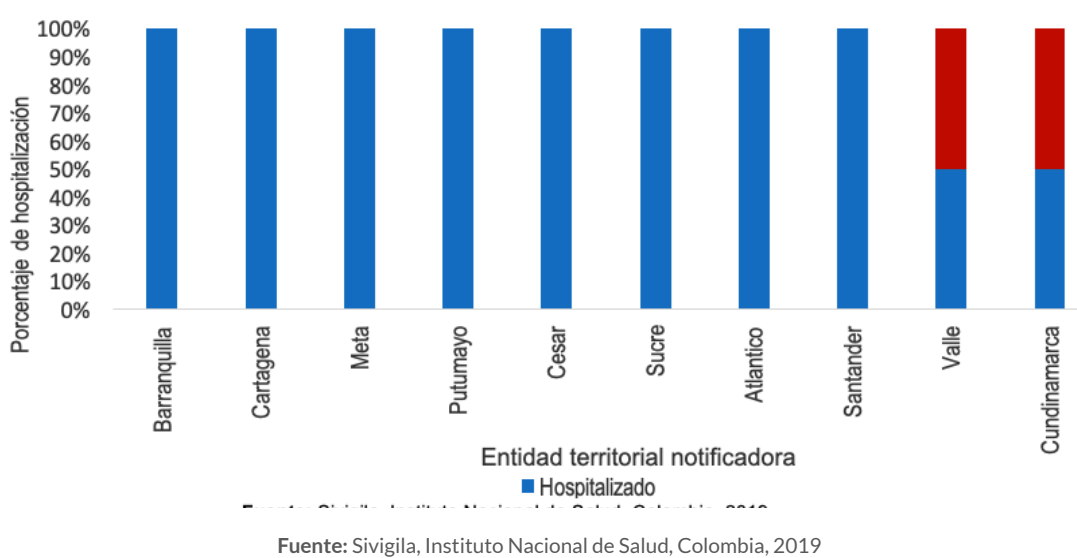

La incidencia nacional de dengue es de 2,3 casos por 100000 habitantes en riesgo. Putumayo, Magdalena, Arauca, Norte de Santander, Cesar, Guaviare, Boyacá y Tolima registran las mayores incidencias (gráfica 13).

\section{Gráfica 13}

Incidencia de dengue por entidad territorial de procedencia, Colombia, semanas epidemiológicas 01, 2019

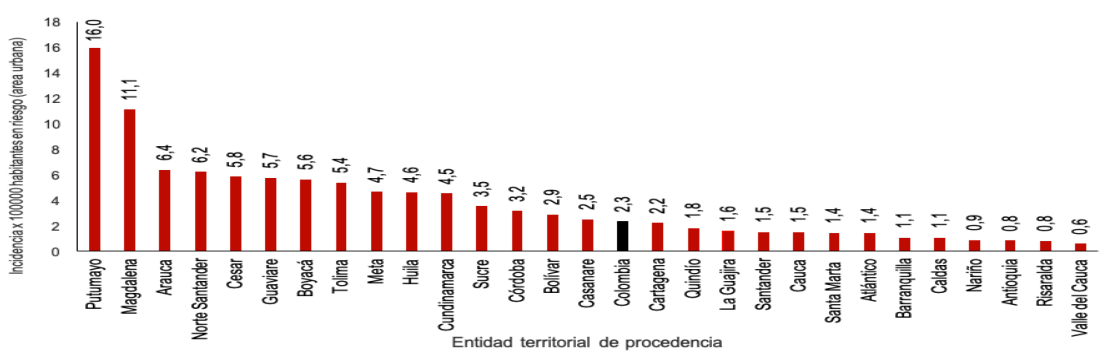

Tema central

Situación $\mathrm{Nal}$

Mortalidad

Trazadores

Sarampión

Brotes

Tablas 
El canal endémico de dengue se ubicó por debajo del límite inferior histórico (2009-2018) en la semana epidemiológicas 1 (gráfica 14).

\section{Gráfica 14}

Canal endémico nacional de dengue, Colombia, semanas epidemiológicas 01, 2019

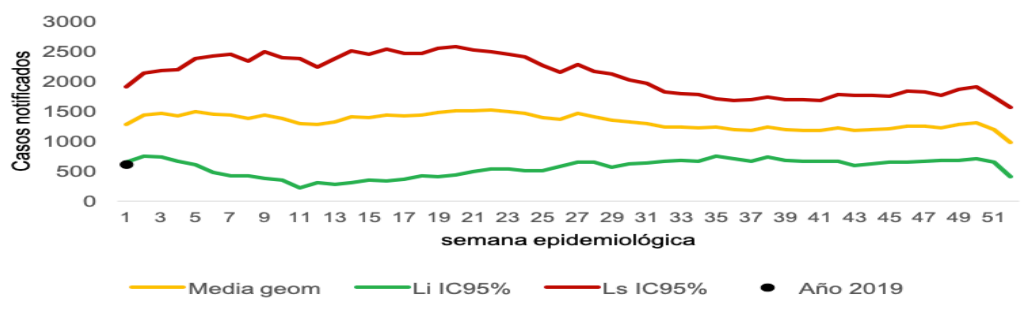

Fuente: Sivigila, Instituto Nacional de Salud, Colombia, 2019

De acuerdo con la situación epidemiológica por departamento de procedencia, cuatro entidades territoriales se encuentran por debajo del número esperado de casos, 25 entidades con un comportamiento dentro de lo esperado, seis entidades territoriales en situación de alarma y una entidad territorial con casos por encima del valor esperado para la primera semana del año comparado con sus comportamiento histórico (2010 a 2018) (tabla 7).

Tabla 7

Comportamiento epidemiológico de dengue, Colombia, semana epidemiológica 1, 2019

\begin{tabular}{|c|c|c|c|c|c|c|c|}
\hline \multirow{2}{*}{$\begin{array}{c}\text { Por debajo del } \\
\text { número esperado } \\
\text { de casos } \\
\text { Valle del Cauca }\end{array}$} & \multicolumn{3}{|c|}{ Dentro del número esperado de casos } & \multicolumn{2}{|c|}{ En situación de alerta } & \multicolumn{2}{|c|}{$\begin{array}{l}\text { Por encima del número } \\
\text { esperado de casos }\end{array}$} \\
\hline & Tolima & Atlántico & Boyacá & San Andres & Putumayo & Magdalena & Guaviare \\
\hline Quindío & Risaralda & La Guajira & Barranquilla & Sucre & Cesar & & Bolivar \\
\hline Caquetá & Amazonas & Vaupés & Cundinamarca & $\begin{array}{l}\text { Norte de } \\
\text { Santander }\end{array}$ & Arauca & & Cesar \\
\hline \multirow[t]{3}{*}{ Huila } & Santander & Choco & Meta & Casanare & Cordoba & & La Guajira \\
\hline & Guainía & Caldas & Vichada & Nariño & Cartagena & & Atlántico \\
\hline & Antioquia & Buenaventura & Cauca & Santa Marta & Bolivar & & Guaviare \\
\hline
\end{tabular}

Fuente: Sivigila, Instituto Nacional de Salud, Colombia, 2019
En la semana epidemiológica 01 se notificaron cinco muertes probables por dengue procedentes de: Galapa (Atlántico), Santa Rosa (Bolívar), Girardot (Cundinamarca), Floridablanca (Santander) y el distrito de Barranquilla, con un caso cada uno.

\section{Malaria}

En la semana epidemiológica 01 de 2019, han ingresado al Sivigila 360 casos de malaria, 351 casos de malaria no complicada y 9 casos de malaria complicada. Para la semana epidemiológica 01 de 2019 , se observa un incremento en la notificación de casos de malaria en las entidades territoriales de Arauca,Caquetá y Norte de Santander en comparación con el promedio de notificación de los años 2012 a 2018 (gráfica 15).

\section{Gráfica 15}

Comparación de la notificación de malaria por entidad territorial de notificación, semana epidemiológica 1 de 2019 con su comportamiento histórico,

$$
\text { Colombia, 2012-2017 }
$$

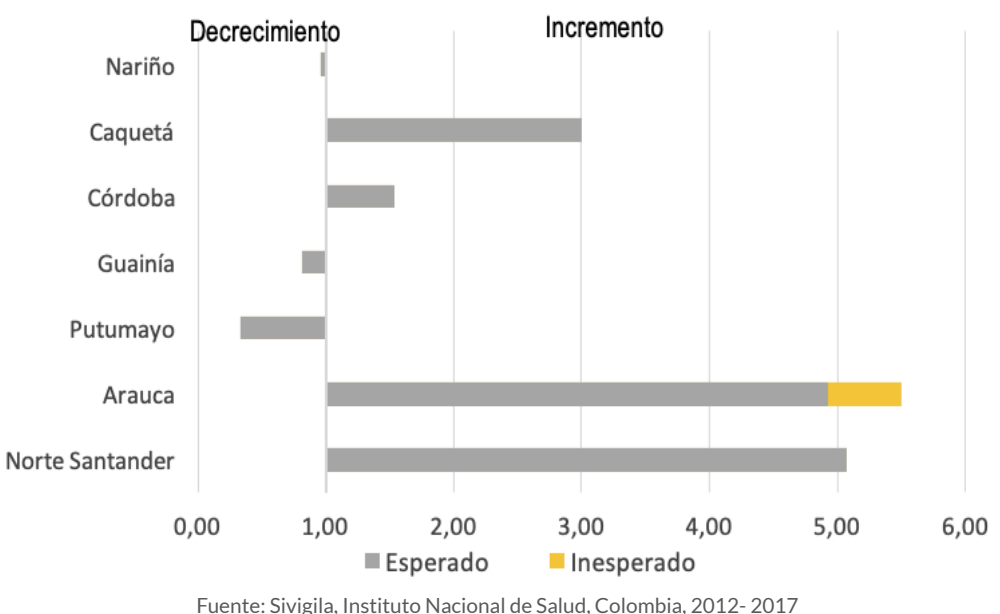

Por especies parasitarias circulantes en el país, hay predominio de infección por P. vivax con 191 casos (el 53,1 \%) P., seguido por falciparum con 163 casos (el $45,3 \%$ ) y 6 casos (el $1,7 \%$ ) corresponden a infección mixta (P. falciparum y P. vivax) (gráfica 16).
Tema central

Situación $\mathrm{Nal}$

Mortalidad

Trazadores

Sarampión

Brotes

Tablas 


\section{Gráfica 16}

Casos notificados de malaria por especie parasitaria, Colombia, semanas epidemiológicas 01, 2019

\section{Malaria asociada (mixta) $\quad \mathbf{I 1 , 7 \%}$}

Malaria P.falciparum

Malaria P.vivax

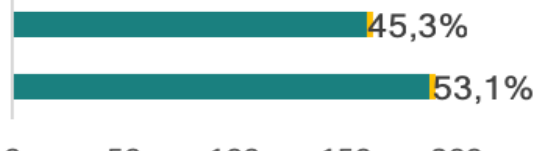

$\begin{array}{llllll}0 & 50 & 100 & 150 & 200 & 250\end{array}$

- No complicada

Fuente: Sivigila, Instituto Nacional de Salud, Colombia, 2019

\section{Malaria no complicada}

Por procedencia Chocó, Córdoba, Nariño, Antioquia, Amazonas, Cauca Y Guainia registraron el $90 \%$ de los casos de malaria no complicada; Choco registra el $20,8 \%$ de todos los casos (tabla 8 ).

$$
\text { Tabla } 8
$$

Casos notificados de malaria no complicada por entidad territorial de procedencia, Colombia semanas epidemiológicas 01, 2019

\begin{tabular}{|c|c|c|c|c|c|c|}
\hline Entidad territorial & $\begin{array}{c}\text { Malaria } \\
\text { mixta }\end{array}$ & $\begin{array}{l}\text { Malaria P. } \\
\text { falciparum }\end{array}$ & $\begin{array}{c}\text { Malaria P. } \\
\text { malarie }\end{array}$ & $\begin{array}{c}\text { Malaria P. } \\
\text { vivax }\end{array}$ & Casos & $\%$ \\
\hline Chocó & 2 & 47 & 0 & 24 & 73 & 20,8 \\
\hline Córdoba & 0 & 12 & 0 & 45 & 57 & 16,2 \\
\hline Nariño & 0 & 53 & 0 & 1 & 54 & 15,4 \\
\hline Antioquia & 1 & 9 & 0 & 27 & 37 & 10,5 \\
\hline Amazonas & 0 & 0 & 0 & 35 & 35 & 10,0 \\
\hline Cauca & 0 & 28 & 0 & 1 & 29 & 8,3 \\
\hline Guainía & 0 & 2 & 0 & 17 & 19 & 5,4 \\
\hline Bolívar & 1 & 6 & 0 & 5 & 12 & 3,4 \\
\hline Norte de Santander & 0 & 0 & 0 & 5 & 5 & 1,4 \\
\hline Caquetá & 0 & 0 & 0 & 3 & 3 & 0,9 \\
\hline Vichada & 0 & 0 & 0 & 2 & 2 & 0,6 \\
\hline Arauca & 0 & 0 & 0 & 2 & 2 & 0,6 \\
\hline Valle del Cauca & 0 & 0 & 0 & 2 & 2 & 0,6 \\
\hline Guaviare & 0 & 0 & 0 & 1 & 1 & 0,3 \\
\hline Buenaventura & 0 & 0 & 0 & 1 & 1 & 0,3 \\
\hline Vaupés & 0 & 1 & 0 & 0 & 1 & 0,3 \\
\hline Meta & 0 & 0 & 0 & 1 & 1 & 0,3 \\
\hline Sucre & 0 & 0 & 0 & 1 & 1 & 0,3 \\
\hline Exterior & 1 & 1 & 0 & 14 & 16 & 4,40 \\
\hline Total & 5 & 159 & 0 & 187 & 351 & 100 \\
\hline
\end{tabular}

Fuente: Sivigila, Instituto Nacional de Salud, Colombia, 2019
El canal endémico de malaria por $P$. falciparum ha estado en situación de seguridad (gráfica 17).

\section{Gráfica 17}

Canal endémico de malaria no complicada por P. falciparum, Colombia, semanas epidemiológicas 01, 2019

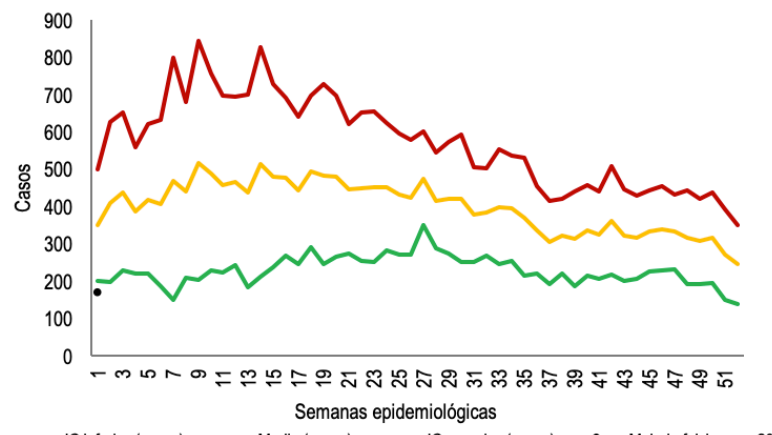

El canal endémico de malaria por P. vivax ha estado en situación de seguridad (gráfica 18).

\section{Gráfica 18}

Canal endémico de malaria no complicada por P. vivax,

Colombia semana epidemiológica 01, 2019

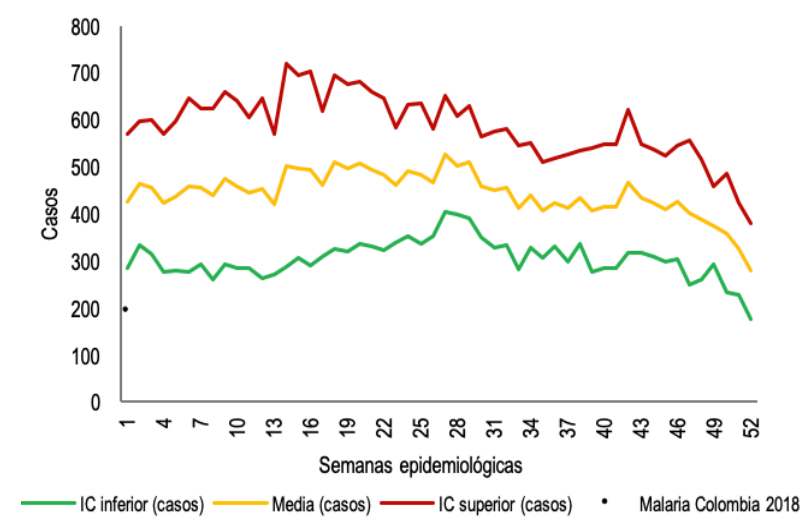

Fuente: Sivigila, Instituto Nacional de Salud, Colombia 2019
Tema centra

Situación Nal.

Mortalidad

Trazadores

Sarampión

Brotes

Tablas 
El riesgo epidemiológico de malaria hasta la semana epidemiológica 01 de 2019 fue de 0,04 casos por cada 1000 habitantes en riesgo (población del área rural), con predominio de infección por $P$. vivax con un IVA de 0,02 casos por cada 1000 habitantes en riesgo (tabla 9).

Tabla 9

Municipios en mayor riesgo epidemiológico para malaria, Colombia semana epidemiológica 01, 2019

\begin{tabular}{|c|c|c|c|c|}
\hline Municipio & Departamento & IPA & IVA & IFA \\
\hline La Pedrera & Amazonas & 4,8 & 4,8 & 0,0 \\
\hline Vigía del Fuerte & Antioquia & 2,3 & 1,7 & 0,6 \\
\hline Puerto Santander & Amazonas & 1,3 & 1,3 & 0,0 \\
\hline Sipí & Chocó & 0,9 & 0,0 & 0,9 \\
\hline Inírida & Guainía & 0,9 & 0,8 & 0,0 \\
\hline Bagadó & Chocó & 0,9 & 0,3 & 0,6 \\
\hline Tadó & Chocó & 0,8 & 0,2 & 0,6 \\
\hline Bajo Baudó & Chocó & 0,8 & 0,5 & 0,3 \\
\hline Tarapacá & Amazonas & 0,7 & 0,7 & 0,0 \\
\hline Miriti - Paraná (ANM) & Amazonas & 0,7 & 0,7 & 0,0 \\
\hline Olaya Herrera & Nariño & 0,6 & 0,0 & 0,6 \\
\hline Segovia & Antioquia & 0,6 & 0,3 & 0,1 \\
\hline Guapi & Cauca & 0,6 & 0,0 & 0,6 \\
\hline Río Quito & Chocó & 0,5 & 0,1 & 0,3 \\
\hline Timbiquí & Cauca & 0,5 & 0,0 & 0,5 \\
\hline Montecristo & Bolívar & 0,5 & 0,2 & 0,3 \\
\hline Tiquisio & Bolívar & 0,4 & 0,1 & 0,2 \\
\hline Norosí & Bolívar & 0,3 & 0,3 & 0,0 \\
\hline
\end{tabular}

Fuente: Sivigila, Instituto Nacional de Salud, Colombia, 2019 - IPA: Número de casos/población a riesgo (área rural)* 1000

\section{Malaria Complicada}

Se notificaron 954 casos de malaria complicada procedentes de 25 entidades territoriales y 92 casos del total son procedentes del exterior (gráfica 19).

\section{Gráfica 19}

Casos notificados de malaria complicada por departamento de procedencia, Colombia, semanas epidemiológicas 01, 2019

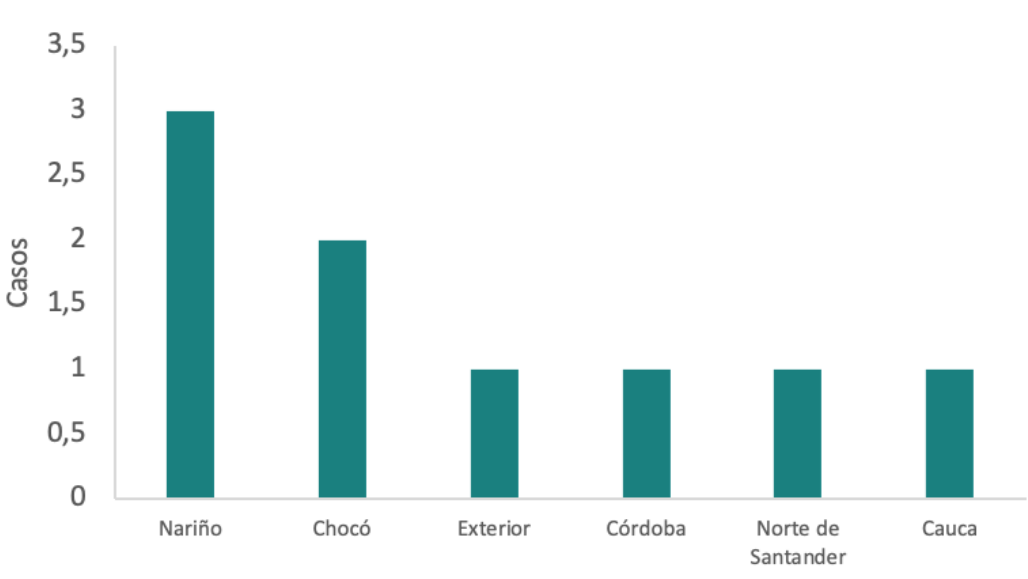

Entidad Territorial de procedencia

Fuente: Sivigila, Instituto Nacional de Salud, Colombia 2019

El 88,9\% de los casos de malaria complicada se registró en el sexo masculino; el 33,3\% de los casos se registró en el grupo de edad de 20 a 24 años; por pertenencia étnica 1 caso (el 11,1\%) se notificaron en indígenas y 4 casos (el 44,4 \%) se notificaron en afrocolombianos; por procedencia, el 22,2 \% de los casos se registró en rural disperso y el $66,7 \%$ pertenecen al régimen subsidiado

De los 9 casos de malaria complicada, 8 casos (el $89 \%$ ) presentaron complicaciones hematológicas y 1 caso (el 11\%) presentaron otras complicaciones (gráfica 20). 


\section{Gráfica 20}

Complicaciones notificadas en los casos de malaria complicada por departamento de procedencia, Colombia, semanas epidemiológicas 01, 2019

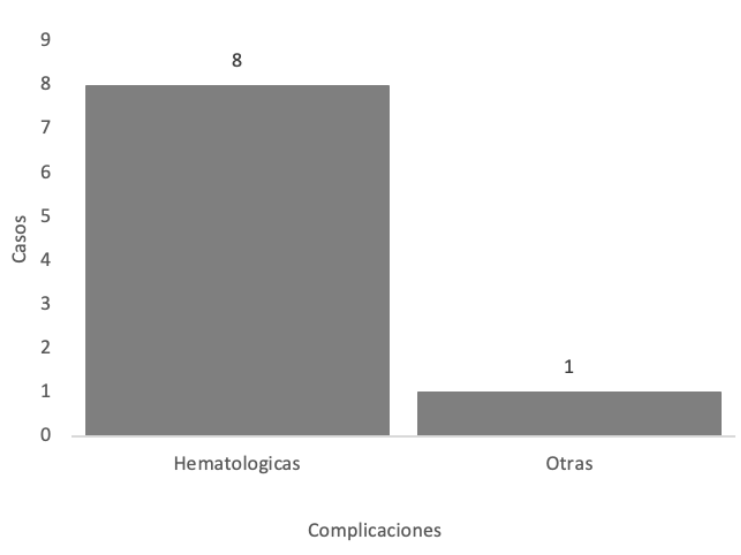

Fuente: Sivigila, Instituto Nacional de Salud, Colombia 2019

\begin{tabular}{l} 
Tema central \\
Situación Nal. \\
Mortalidad \\
\hline Trazadores \\
Sarampión \\
\hline Brotes \\
\hline Tablas
\end{tabular}

\section{${ }^{*}$ Casos registrados como procedentes del exterior}

Hasta la semana epidemiológica 01 del 2019, se han notificado 1.7 casos procedentes del exterior, 16 casos de malaria no complicada y 1 caso de malaria complicada; 15 casos se han registrado con infección por P. vivax, 1 caso con infección por P. falciparum y 1 caso con infección mixta (P. falciparum y P. vivax) (tabla 10).

Tabla 10

Casos notificados de malaria procedentes del exterior Colombia, semanas epidemiológicas 01, 2019

\begin{tabular}{ccccccc} 
País de procedencia & $\begin{array}{c}\text { Malaria } \\
\text { mixta }\end{array}$ & $\begin{array}{c}\text { Malaria P. } \\
\text { falciparum }\end{array}$ & $\begin{array}{c}\text { Malaria P. } \\
\text { vivax }\end{array}$ & Casos & $\%$ \\
\hline Venezuela & 1 & 1 & 15 & 17 & 100 \\
\hline Total & 1 & 1 & 15 & 17 & \\
\hline
\end{tabular}

Fuente: Sivigila, Instituto Nacional de Salud, Colombia 2019 


\section{BROTES Y SITUACIONES DE EMERGENCIA EN SALUD PÚBLICA}

\section{ALERTAS INTERNACIONALES}

\section{Fiebre amarilla, Nigeria}

El 22 de noviembre de 2018, la Organización Mundial de la Salud fue informada de un grupo de casos sospechosos de fiebre amarilla y muertes en el estado de Edo, Nigeria. El estado de Edo se ubica a menos de $400 \mathrm{~km}$ de Lagos en un denso eje de movimiento de población entre Lagos y el sureste de Nigeria. El estado de Edo también es un área endémica conocida para la fiebre de Lassa, que inicialmente se sospechó que causó el brote. Desde el 22 de septiembre hasta el 31 de diciembre de 2018, se han notificado un total de 146 casos sospechosos, 42 presuntos positivos y 32 casos confirmados, incluidas 26 muertes (tasa de letalidad presunta: 18\%) en 15 de las 18 Áreas de Gobierno Local (APG) en Estado Edo.

Los hombres representan la mayoría de los casos (108 de 146, 74\%), y el grupo de edad más afectado es de 10 a 19 años con 48 casos (33\%), seguido del grupo de edad de 20 a 29 años con 36 casos (25\%). Inicialmente, las LGA afectadas eran rurales, pero desde fines de noviembre de 2018, se han informado casos sospechosos de tres LGA urbanas.

En la actualidad, existe un riesgo moderado a nivel regional debido al posible movimiento de los individuos de los estados afectados a áreas adyacentes y países vecinos, y especialmente si hay visitantes no vacunados durante la temporada festiva de fin de año. El riesgo global actual es bajo a nivel global.

Nigeria se enfrenta a varias emergencias de salud pública concurrentes, como cólera, poliovirus circulantes derivados de la vacuna, viruela del mono, sarampión y brotes de fiebre de Lassa en otros estados, y una crisis humanitaria en el noreste del país.

\section{Poliomielitis, República Democrática del Congo}

A partir de octubre de 2018, se detectaron aislamientos de poliovirus de tipo 2 (cVDPV2) derivados de la vacuna, vinculados genéticamente, en dos casos de la provincia de Haut-Katanga (distrito de Mufunga-Sampwe) en la República Democrática del Congo. El primer caso fue un niño de 11 años que tuvo inicio de parálisis flácida aguda (PFA) el 6 de octubre. El segundo caso fue un niño de 29 meses de edad que presentó síntomas el 7 de octubre y es un contacto conocido del primer caso. Los virus aislados son una nueva aparición y no están relacionados con los cVDPV2 detectados previamente que afectan al país. Este es el cuarto brote distinto de cVDPV2 detectado en el país desde junio de 2017. En total, se han confirmado 42 casos de cVDPV2 desde la detección del primer brote en junio de 2017, 20 de los cuales se detectaron en 2018.

En febrero de 2018, el gobierno declaró que cVDPV2 era una emergencia nacional de salud pública. El 26 de julio de 2018, el Ministro de Salud, el Director General de la OMS, el Director Regional para África y los gobernadores provinciales convocaron una reunión urgente de alto nivel y firmaron la 'Declaración de Kinshasa para la erradicación de la poliomielitis'.

Actualmente, la OMS considera que el riesgo general para la salud pública nacional asociado con estos cuatro brotes es muy alto. El riesgo de propagación internacional, en particular a los países vecinos también sigue siendo alto debido a la continuación de estos brotes cerca de las fronteras internacionales. Este riesgo se ve magnificado por los movimientos de población conocidos entre las áreas afectadas de la República Democrática del Congo, Uganda, República Centroafricana, Zambia y Sudán del Sur.

Fuente: https://www.who.int/csr/don/08-january-2019-poliovirusdrc/en/

Fuente: https://www.who.int/csr/don/09-january-2019-yellow-fever-nigeria/en/ 


\section{Dengue en Colombia a semana epidemiológica 52 de 2018}

Se confirma un aumento en la notificación de casos de dengue a partir de la semana epidemiológica 42 comprometiendo 104 municipios de 25 entidades territoriales; con mayor circulación de dengue tipo 1.

Con cortea29 de diciembre, sehannotificadoal sistema de información 44.825 casos de dengue; 21.242 casos sin signos de alarma (47,4\%); 23.057 casos con signos de alarma $(51,4 \%)$ y 526 casos de dengue grave $(1,2 \%)$.

Según el comportamiento epidemiológico las entidades territoriales de Norte de Santander, Córdoba, Santa Marta, Magdalena, Cartagena, Meta, Guaviare, Bolívar, Cesar, La Guajira, Atlántico y Guaviare superan la notificación esperada.

El indicador de hospitalización para dengue grave es de $93.9 \%$, la incidencia nacional de dengue es de 172,9 casos por 100000 habitantes en riesgo y la letalidad por dengue es de $0,05 \%$ y la letalidad por dengue grave es de 4,3\%, habiendose notificado 176 muertes, siendo 23 confirmados, 70 descartados y 83 en estudio.

Recomendaciones para las entidades territoriales: deben fortalecerse las acciones de todos los componentes de la estrategia de gestión integral de dengue, incluyendo la inteligencia epidemiológica que permita abordar y focalizar los sitios de intervención a corto plazo, disminuyendo la cadena de transmisión. Esta acción debe ir acompañada de la modificación de los entornos y el fortalecimiento de la capacidad local para la prevención de casos, vigilancia en Salud Pública, Control sanitario y atención integral de casos mediante la consolidación y conformación de equipos funcionales y de alerta y respuesta inmediata. Evento en seguimiento.

$$
\text { Fuente: grupo ETV zonosis, INS }
$$




\section{EVENTOS TRAZADORES}

Hasta la semana epidemiológica 52 de 2018 ingresaron a la vigilancia integrada de Sarampión y Rubeola 7102 casos sospechosos, de los cuales se han descartado 6488, permanecen en estudio 416 casos; en la Semana epidemiológica 01 de 2019 han ingresado 37 casos sospechosos de Sarampión, de los cuales 34 permanecen en estudio y tres se han confirmado.

Hasta el 11 de Enero de 2019, el Instituto Nacional de Salud ha confirmado 201 casos de sarampión, de los cuales 54 casos son importados, 113 casos relacionados con la importación (35 casos de transmisión secundaria en personas procedentes de Venezuela y 78 casos relacionados con la importación en colombianos) y 34 con fuente de infección en investigación.

\begin{tabular}{|c|c|c|c|c|c|c|}
\hline \multirow[t]{2}{*}{$\begin{array}{l}\text { Departamento de } \\
\text { Procedencia }\end{array}$} & \multicolumn{2}{|c|}{$\begin{array}{l}\text { Sarampión y Rubeola a Semana } \\
\text { epidemiólogica } 52 \text { de } 2018\end{array}$} & \multirow[t]{2}{*}{$\begin{array}{l}\text { Total } \\
\text { general }\end{array}$} & $\begin{array}{r}\text { Sarampión y } \\
\text { Semana epide } \\
\text { de } 2\end{array}$ & $\begin{array}{l}\text { ubeola a } \\
\text { iólogica } 01 \\
9\end{array}$ & \multirow[t]{2}{*}{$\begin{array}{l}\text { Total } \\
\text { general }\end{array}$} \\
\hline & Confirmado & En estudio & & Confirmado & En estudio & \\
\hline
\end{tabular}

\begin{tabular}{|c|c|c|c|c|c|c|c|}
\hline AMAZONAS & & 6 & 3 & 9 & & & 0 \\
\hline ANTIOQUIA & 2 & 591 & 84 & 677 & & 1 & 1 \\
\hline ARAUCA & 1 & 10 & & 11 & & & 0 \\
\hline ATLANTICO & 3 & 85 & 7 & 95 & & & 0 \\
\hline BARRANQUILLA & 33 & 196 & 48 & 277 & & 3 & 3 \\
\hline BOGOTA & 5 & 3613 & 104 & 3722 & 1 & 10 & 11 \\
\hline BOLIVAR & 12 & 48 & 7 & 67 & & & 0 \\
\hline BOYACA & & 66 & & 66 & & & 0 \\
\hline CALDAS & & 51 & 2 & 53 & & & 0 \\
\hline CAQUETA & & 18 & & 18 & & & 0 \\
\hline CARTAGENA & 67 & 355 & 74 & 496 & & 5 & 5 \\
\hline CASANARE & & 7 & 1 & 8 & & & 0 \\
\hline CAUCA & 1 & 23 & 4 & 28 & & & 0 \\
\hline CESAR & 3 & 87 & 4 & 94 & & 1 & 1 \\
\hline $\mathrm{CHOCO}$ & & 5 & & 5 & & & 0 \\
\hline CORDOBA & & 20 & 3 & 23 & & & 0 \\
\hline CUNDINAMARCA & 2 & 330 & 14 & 346 & & 2 & 2 \\
\hline GUAINIA & & 2 & & 2 & & & 0 \\
\hline GUAJIRA & & 17 & 1 & 18 & & 1 & 1 \\
\hline GUAVIARE & & 4 & & 4 & & & 0 \\
\hline HUILA & & 28 & & 28 & & & 0 \\
\hline MAGDALENA & 1 & 24 & & 25 & & & 0 \\
\hline META & & 68 & 3 & 71 & & 1 & 1 \\
\hline NARIÑO & & 58 & 5 & 63 & & & 0 \\
\hline NORTE SANTANDER & 7 & 173 & 11 & 191 & & 3 & 3 \\
\hline PUTUMAYO & & 8 & & 8 & & & 0 \\
\hline QUINDIO & & 45 & & 45 & & & 0 \\
\hline RISARALDA & & 103 & 7 & 110 & & & 0 \\
\hline SAN ANDRES & & 23 & & 23 & & & 0 \\
\hline SANTANDER & & 66 & 6 & 72 & & & 0 \\
\hline STA MARTA D.E. & & 30 & 4 & 34 & & & 0 \\
\hline SUCRE & 7 & 112 & 1 & 120 & & 1 & 1 \\
\hline TOLIMA & & 46 & 6 & 52 & & 1 & 1 \\
\hline VALLE & & 77 & 4 & 81 & & 1 & 1 \\
\hline VAUPES & & 1 & & 1 & & & 0 \\
\hline ZEXTERIOR & 54 & 92 & 13 & 159 & 2 & 4 & 6 \\
\hline Total general & 198 & 6488 & 416 & 7102 & 3 & 34 & 37 \\
\hline
\end{tabular}

La información es notificada semanalmente por las entidades territoriales (ET) al Instituto Nacional de Salud (INS) a través del Sistema de vigilancia en salud pública (Sivigila). El número de casos, puede variar después de que se realizan unidades de análisis en las ET para el ajuste y la clasificación de caso en cada evento.
Tema centra

Situación Nal.

Mortalidad

Trazadores

Sarampión

Brotes

Tablas 


\section{TABLAS DE MANDO NACIONAL}

\begin{tabular}{|c|c|c|c|c|c|c|c|c|c|c|c|c|c|c|c|c|c|c|c|c|c|}
\hline \multirow[b]{2}{*}{ Departamento } & \multicolumn{3}{|c|}{ Accidente ofidico } & \multicolumn{3}{|c|}{$\begin{array}{c}\text { Agresiones por animales } \\
\text { potencialmente transmisores de } \\
\text { rabia }\end{array}$} & \multicolumn{3}{|c|}{ Dengue } & \multicolumn{3}{|c|}{ ESAVI Grave } & \multicolumn{3}{|c|}{ Intento de suicidio } & \multicolumn{3}{|c|}{$\begin{array}{l}\text { Intoxicaciones por sustancias } \\
\text { quimicas }\end{array}$} & \multicolumn{3}{|c|}{ IRAG inusitado } \\
\hline & 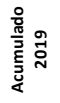 & 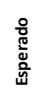 & 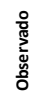 & 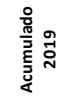 & $\begin{array}{l}\frac{0}{0} \\
\frac{0}{0} \\
\frac{0}{4} \\
\frac{4}{4}\end{array}$ & 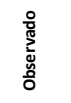 & 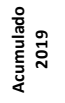 & $\begin{array}{l}\text { 음 } \\
\frac{0}{0} \\
\text { s. }\end{array}$ & 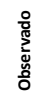 & 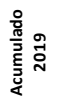 & $\begin{array}{l}\frac{0}{0} \\
\frac{0}{0} \\
\frac{0}{4}\end{array}$ & 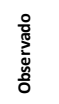 & 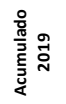 & $\begin{array}{l}\frac{0}{0} \\
\frac{0}{0} \\
\frac{5}{4}\end{array}$ & 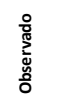 & 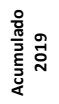 & 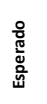 & 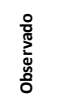 & 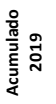 & 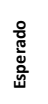 & 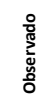 \\
\hline Amazonas & 0 & 1 & 0 & 2 & 4 & 2 & 0 & 3 & 0 & 0 & 1 & 0 & 0 & 1 & 0 & 0 & 0 & 0 & 0 & 0 & 0 \\
\hline Antioquia & 11 & 13 & 11 & 230 & 231 & 230 & 39 & 136 & 39 & 0 & 3 & 2 & 58 & 73 & 58 & 29 & 40 & 29 & 2 & 16 & 16 \\
\hline Arauca & 1 & 3 & 1 & 12 & 12 & 12 & 11 & 9 & 11 & 0 & 1 & 0 & 2 & 4 & 2 & 2 & 3 & 2 & 0 & 1 & 2 \\
\hline Atlantico & 1 & 2 & 1 & 41 & 35 & 41 & 17 & 46 & 17 & 0 & 1 & 1 & 12 & 12 & 12 & 6 & 8 & 6 & 0 & 4 & 0 \\
\hline Barranquilla & 0 & 0 & 0 & 35 & 34 & 35 & 13 & 51 & 13 & 0 & 1 & 0 & 7 & 14 & 7 & 10 & 14 & 10 & 0 & 3 & 0 \\
\hline Bogota & 0 & 0 & 0 & 178 & 339 & 178 & 0 & 0 & 0 & 1 & 7 & 11 & 4 & 18 & 4 & 21 & 54 & 21 & 2 & 10 & 13 \\
\hline Bolivar & 7 & 4 & 7 & 30 & 31 & 30 & 20 & 24 & 20 & 0 & 2 & 0 & 8 & 7 & 8 & 6 & 6 & 6 & 1 & 1 & 1 \\
\hline Boyaca & 0 & 1 & 0 & 96 & 87 & 96 & 5 & 10 & 5 & 0 & 2 & 1 & 9 & 12 & 9 & 6 & 7 & 6 & 1 & 1 & 3 \\
\hline Buenaventura & 0 & 1 & 0 & 2 & 2 & 2 & 0 & 2 & 0 & 0 & 0 & 0 & 0 & 0 & 0 & 0 & 1 & 0 & 0 & 0 & 0 \\
\hline Caldas & 0 & 1 & 0 & 45 & 61 & 45 & 3 & 10 & 3 & 0 & 1 & 0 & 15 & 17 & 15 & 18 & 11 & 18 & 0 & 2 & 4 \\
\hline Caqueta & 1 & 4 & 1 & 14 & 13 & 14 & 0 & 19 & 0 & 0 & 1 & 0 & 10 & 6 & 10 & 1 & 4 & 1 & 0 & 0 & 0 \\
\hline $\begin{array}{l}\text { Cartagena } \\
\end{array}$ & 0 & 1 & 0 & 8 & 12 & 8 & 22 & 18 & 22 & 0 & 2 & 0 & 1 & 6 & 1 & 13 & 11 & 13 & 0 & 1 & 0 \\
\hline Casanare & 1 & 4 & 1 & 21 & 23 & 21 & 7 & 22 & 7 & 0 & 1 & 1 & 7 & 6 & 7 & 3 & 2 & 3 & 0 & 0 & 0 \\
\hline Cauca & 4 & 3 & 4 & 75 & 89 & 75 & 4 & 9 & 4 & 0 & 1 & 1 & 17 & 16 & 17 & 3 & 7 & 3 & 0 & 2 & 0 \\
\hline $\begin{array}{l}\text { Cesar } \\
\text { Shoct }\end{array}$ & $\frac{4}{5}$ & 4 & 4 & 33 & 27 & 33 & 47 & 50 & 47 & 0 & 1 & 0 & 11 & 10 & 11 & 2 & 12 & 2 & 0 & 4 & 0 \\
\hline Choco & 5 & 4 & 5 & $\begin{array}{ll}4 \\
51\end{array}$ & 2 & 4 & 0 & 3 & 0 & 0 & 1 & 1 & 0 & 1 & 0 & 2 & 3 & 2 & 0 & 0 & 0 \\
\hline Cordoba & 1 & 5 & 1 & 51 & 48 & 51 & 30 & 37 & 30 & 0 & 1 & 1 & 13 & 15 & 13 & 7 & 11 & 7 & 0 & 2 & 0 \\
\hline $\begin{array}{l}\text { Cundinamarca } \\
\text { Guainia }\end{array}$ & $\frac{0}{0}$ & 1 & 0 & $\frac{211}{0}$ & $\frac{169}{1}$ & 211 & $\frac{20}{0}$ & 69 & $\frac{20}{0}$ & 0 & 2 & 2 & 27 & 29 & 27 & 8 & 9 & 8 & 0 & 2 & 1 \\
\hline $\begin{array}{l}\text { Guainia } \\
\text { GGaijara }\end{array}$ & 0 & 0 & 0 & 0 & 1 & 0 & 0 & 1 & 0 & 0 & 1 & 0 & 0 & 0 & $\frac{0}{3}$ & 0 & 0 & 0 & 0 & 0 & 0 \\
\hline $\begin{array}{l}\text { Guaalira } \\
\text { Guaviare }\end{array}$ & 3 & 2 & $\frac{0}{3}$ & 17 & $\frac{18}{3}$ & 17 & $\frac{9}{4}$ & $\frac{23}{4}$ & $\frac{9}{4}$ & 0 & 1 & 0 & 3 & 4 & 3 & 0 & 4 & $\frac{0}{2}$ & 0 & $\frac{1}{1}$ & $\frac{0}{0}$ \\
\hline Huila & 1 & 1 & 1 & $\frac{1}{72}$ & 58 & $\frac{1}{72}$ & 4 & $\begin{array}{l}4 \\
93 \\
\end{array}$ & $\frac{4}{33}$ & $\frac{0}{0}$ & $\frac{1}{3}$ & $\frac{0}{0}$ & $\frac{1}{14}$ & $\frac{1}{15}$ & $\frac{1}{14}$ & $\frac{2}{10}$ & 0 & $\frac{2}{10}$ & 0 & $\frac{1}{3}$ & $\frac{0}{3}$ \\
\hline Magdalena & 2 & 2 & 2 & 32 & 25 & 32 & 53 & 13 & 53 & 0 & 1 & 0 & $\frac{14}{5}$ & $\frac{15}{6}$ & $\frac{\frac{14}{5}}{5}$ & $\frac{10}{5}$ & $\frac{10}{6}$ & $\frac{10}{5}$ & $\frac{1}{0}$ & 3 & $\frac{3}{0}$ \\
\hline Meta & 4 & 2 & 4 & 57 & 52 & 57 & 36 & 76 & 36 & 0 & 1 & 0 & 9 & 10 & 9 & 11 & 10 & 11 & 0 & 1 & 1 \\
\hline Nariño & 6 & 4 & 6 & 84 & 73 & 84 & 2 & 7 & 2 & 0 & 2 & 2 & 19 & 19 & 19 & 24 & 12 & 24 & 0 & 1 & 0 \\
\hline Norte Santander & 2 & 2 & 2 & 61 & 67 & 61 & 64 & 89 & 64 & 0 & 1 & 2 & 11 & 12 & 11 & 15 & 11 & 15 & 0 & 1 & 1 \\
\hline Putumayo & 4 & 5 & 4 & 18 & 17 & 18 & 25 & 24 & 25 & 0 & 1 & 0 & 10 & $\frac{12}{7}$ & 10 & $\frac{3}{2}$ & $\frac{11}{4}$ & $\frac{3}{2}$ & 0 & 1 & 0 \\
\hline Quindio & 1 & 3 & 1 & 55 & 51 & 55 & 9 & 31 & 9 & 0 & 2 & 0 & 5 & 9 & 5 & 4 & 12 & 4 & 0 & 2 & 2 \\
\hline Risaralda & 0 & 0 & 0 & 26 & 60 & 26 & 6 & 13 & 6 & 0 & 2 & 0 & 9 & 14 & 9 & 1 & 7 & 1 & 0 & 2 & 0 \\
\hline San Andres & 0 & 1 & 0 & 7 & 6 & 7 & 0 & 1 & 0 & 0 & 1 & 0 & 0 & 0 & 0 & 0 & 1 & 0 & 0 & 0 & 0 \\
\hline Santa Marta D.E & 0 & 1 & 0 & 12 & 32 & 12 & 7 & 14 & 7 & 0 & 1 & 0 & 3 & 3 & 3 & 2 & 3 & 2 & 0 & 4 & 1 \\
\hline Santander & 8 & 4 & 8 & 89 & 85 & 89 & 23 & 117 & 23 & 0 & 2 & 1 & 17 & 18 & 17 & 8 & 5 & 8 & 0 & 5 & 3 \\
\hline Sucre & 1 & 2 & 1 & 35 & 34 & 35 & 21 & 36 & 21 & 0 & 2 & 0 & 6 & 4 & 6 & 8 & 6 & 8 & 0 & 2 & 0 \\
\hline Tolima & 0 & 2 & 0 & 110 & 86 & 110 & 52 & 167 & 52 & 0 & 2 & 2 & 10 & 16 & 10 & 10 & 8 & 10 & 0 & 1 & 0 \\
\hline Valle & 0 & 1 & 0 & 183 & 199 & 183 & 22 & 222 & 22 & 0 & 3 & 3 & 40 & 42 & 40 & 20 & 24 & 20 & 3 & 6 & 7 \\
\hline Vaupes & 2 & 1 & 2 & 0 & 2 & 0 & 0 & 0 & 0 & 0 & 1 & 0 & 0 & 1 & 0 & 0 & 0 & 0 & 0 & 0 & 0 \\
\hline Vichada & 0 & 1 & 0 & 2 & 2 & 2 & 0 & 2 & 0 & 0 & 1 & 0 & 1 & 0 & 1 & 1 & 0 & 1 & 0 & 1 & 0 \\
\hline Total nacional & 70 & 87 & 70 & 1949 & 2089 & 1949 & 604 & 1451 & 604 & 1 & 58 & 31 & 364 & 428 & 364 & 260 & 326 & 260 & 10 & 84 & 58 \\
\hline
\end{tabular}

Tema central

Situación Nal.

Mortalidad

Trazadores

Sarampión

Brotes

Tablas $\mathbf{2}_{\substack{\text { INSTITUTO } \\ \text { NALIONAL DE }}}^{\text {SALUD }}$ 


\begin{tabular}{|c|c|c|c|c|c|c|c|c|c|c|c|c|c|c|c|c|c|c|c|c|c|}
\hline \multirow[b]{2}{*}{ Departamento } & \multicolumn{3}{|c|}{ Malaria } & \multicolumn{3}{|c|}{ Meningitis bacteriana } & \multicolumn{3}{|c|}{$\begin{array}{c}\begin{array}{c}\text { Morbilidad por IRA consulta externa } \\
\text { y urgencias }\end{array} \\
\end{array}$} & \multicolumn{3}{|c|}{$\begin{array}{c}\begin{array}{c}\text { Morbilidad por IRA hospitalizaciones } \\
\text { en sala general }\end{array} \\
\end{array}$} & \multicolumn{3}{|c|}{$\begin{array}{c}\text { Morbilidad por IRA hospitalizaciones } \\
\text { en UCI }\end{array}$} & \multicolumn{3}{|c|}{ Morbilidad por EDA } & \multicolumn{3}{|c|}{ Morbilidad Materna Extrema } \\
\hline & 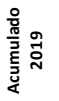 & $\begin{array}{l}\frac{8}{0} \\
\frac{0}{0} \\
\frac{80}{4}\end{array}$ & 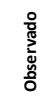 & 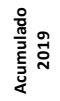 & 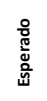 & 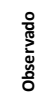 & 总 & 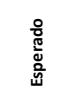 & 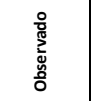 & 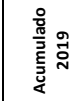 & 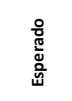 & 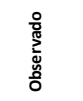 & 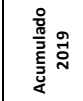 & 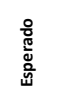 & 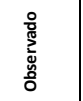 & 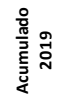 & 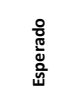 & 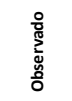 & 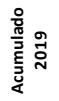 & 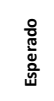 & 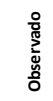 \\
\hline Amazonas & 35 & 62 & 35 & 0 & 2 & 1 & 63 & 692 & 543 & 0 & 8 & 0 & 0 & 0 & 0 & 47 & 133 & 62 & 0 & 2 & 1 \\
\hline Antioquia & 37 & 378 & 37 & 2 & 33 & 32 & 16246 & 70249 & 75107 & 449 & 1961 & 2360 & 11 & 60 & 45 & 7859 & 8104 & 8307 & 5 & 60 & 49 \\
\hline Arauca & 2 & $\begin{array}{ll}4 \\
\end{array}$ & 2 & 0 & 6 & 1 & 304 & 1883 & 1881 & 24 & 85 & 90 & 0 & 4 & 2 & 166 & 190 & 160 & 0 & 4 & 2 \\
\hline Atlantico & 0 & 2 & 0 & 0 & 4 & 10 & 892 & 7635 & 6747 & 24 & 127 & 74 & 8 & 57 & 37 & 422 & 950 & 764 & 4 & 15 & 29 \\
\hline Barranquilla & 0 & 1 & 0 & 0 & 2 & 16 & 2236 & 11437 & 11408 & 26 & 194 & 200 & 6 & 43 & 46 & 811 & 1527 & 1598 & 3 & 17 & 19 \\
\hline Bogota & 0 & 1 & 0 & 0 & 35 & 36 & 24335 & 90825 & 116570 & 640 & 3918 & 3761 & 73 & 353 & 391 & 12442 & 14073 & 13070 & 7 & 78 & 72 \\
\hline Bolivar & 12 & 64 & 12 & 0 & 8 & 8 & 1803 & 9446 & 8933 & 48 & 153 & 221 & 0 & 0 & 2 & 548 & 724 & 584 & 1 & 13 & 11 \\
\hline Boyaca & 0 & 1 & 0 & 0 & 3 & 3 & 2335 & 10537 & 10082 & 38 & 303 & 307 & 0 & 9 & 27 & 822 & 1097 & 1042 & 2 & 12 & 13 \\
\hline Buenaventura & 1 & 29 & 1 & 0 & 3 & 0 & 220 & 1133 & 952 & 0 & 0 & 0 & 0 & 0 & 0 & 80 & 166 & 99 & 0 & 4 & 3 \\
\hline Caldas & 0 & 3 & 0 & 0 & 3 & 3 & 1463 & 9079 & 7565 & 139 & 317 & 539 & 4 & 29 & 16 & 877 & 845 & 741 & 1 & 8 & 5 \\
\hline Caqueta & 3 & 2 & 3 & 0 & 2 & 6 & 425 & 3283 & 2589 & 10 & 116 & 101 & 0 & 1 & 0 & 410 & 545 & 442 & 3 & 7 & 8 \\
\hline Cartagena & 0 & 1 & 0 & 0 & 9 & 4 & 2743 & 13625 & 16722 & 40 & 331 & 310 & 25 & 97 & 81 & 915 & 1051 & 1062 & 3 & 17 & 11 \\
\hline Casanare & 0 & 2 & 0 & 0 & 1 & 2 & 363 & 1834 & 1834 & 11 & 24 & 38 & 0 & 0 & 0 & 223 & 265 & 227 & 0 & 4 & 2 \\
\hline $\begin{array}{l}\text { Cauca } \\
\end{array}$ & 30 & 30 & 30 & 1 & 5 & 8 & 1379 & 8565 & 7190 & 25 & 109 & 95 & 0 & 0 & 0 & 761 & 1260 & 1057 & 0 & 19 & 8 \\
\hline Cesar & 0 & 1 & 0 & 0 & 2 & 6 & 1554 & 10189 & 9405 & 63 & 508 & 367 & 5 & 27 & 26 & 885 & 1071 & 847 & 3 & 19 & 15 \\
\hline Choco & 75 & 547 & 75 & 1 & 1 & 1 & 300 & 1476 & 1672 & 32 & 129 & 125 & 0 & 0 & 2 & 120 & 234 & 224 & 1 & 10 & 5 \\
\hline $\begin{array}{l}\text { Cordoba } \\
\end{array}$ & 58 & 158 & 58 & 0 & 5 & 7 & 2099 & 11501 & 10860 & 32 & 289 & 215 & 4 & 29 & 60 & 797 & 1148 & 871 & 6 & 28 & 35 \\
\hline Cundinamarca & 0 & 2 & 0 & 1 & 8 & 15 & 4835 & 18656 & 21076 & 181 & 533 & 721 & 13 & 30 & 64 & 2883 & 2550 & 2796 & 4 & 25 & 23 \\
\hline Guainia & 19 & 43 & 19 & 0 & 0 & 0 & 39 & 198 & 291 & 13 & 19 & 35 & 0 & 0 & 0 & 33 & 32 & 48 & 0 & 1 & 1 \\
\hline Guajira & 0 & 18 & 0 & 0 & 4 & 1 & 1091 & 9849 & 10423 & 126 & 389 & 389 & 21 & 45 & 84 & 677 & 1215 & 1145 & 6 & 15 & 23 \\
\hline Guaviare & 1 & 30 & 1 & 0 & 0 & 0 & 91 & 560 & 446 & 14 & 46 & 56 & 0 & 0 & 0 & 72 & 70 & 86 & 0 & 1 & 0 \\
\hline Huila & 0 & 1 & 0 & 0 & 3 & 4 & 1616 & 9548 & 7591 & 22 & 242 & 148 & 5 & 28 & 23 & 1220 & 1362 & 1232 & 1 & 13 & 5 \\
\hline Magdalena & 0 & 2 & 0 & 0 & 3 & 7 & 1378 & 6419 & 6672 & 48 & 152 & 197 & 0 & 0 & 0 & 800 & 829 & 771 & 1 & 11 & 11 \\
\hline Meta & 1 & 4 & 1 & 0 & 8 & 2 & 1121 & 5713 & 6024 & 20 & 110 & 123 & 6 & 13 & 38 & 1076 & 1182 & 972 & 0 & 12 & 5 \\
\hline Nariño & 57 & 229 & 57 & 0 & 11 & 7 & 2323 & 14671 & 12027 & 75 & 349 & 265 & 2 & 7 & 7 & 1065 & 1839 & 1551 & 4 & 17 & 16 \\
\hline Norte Santander & 6 & 5 & 6 & 1 & 4 & 10 & 2746 & 12314 & 14433 & 174 & 863 & 908 & 19 & 45 & 82 & 1842 & 1467 & 1904 & 8 & 17 & 24 \\
\hline Putumayo & 0 & 6 & 0 & 0 & 3 & 2 & 235 & 2265 & 1690 & 23 & 90 & 197 & 0 & 0 & 0 & 226 & 313 & 294 & 2 & 4 & 4 \\
\hline Quindio & 0 & 2 & 0 & 0 & 1 & $\frac{1}{2}$ & 1363 & 6200 & 5251 & 74 & 280 & 304 & 1 & 14 & 11 & 731 & 808 & 609 & 2 & 5 & 9 \\
\hline Risaralda & 0 & 19 & 0 & 0 & 4 & 3 & 1989 & 7899 & 7899 & 53 & 240 & 199 & 0 & 8 & 5 & 814 & 1067 & 910 & 1 & 8 & 7 \\
\hline San Andres & 0 & 0 & 0 & 0 & 0 & 0 & 106 & 633 & 464 & 70 & 56 & 80 & 3 & $\frac{1}{20}$ & 3 & 65 & 80 & 70 & 0 & 1 & 2 \\
\hline Santa Marta D.E & 0 & 1 & 0 & 0 & 0 & 2 & 307 & 1686 & 1480 & 14 & 118 & 98 & 7 & 29 & 38 & 269 & 286 & 271 & 0 & 6 & 5 \\
\hline Santander & 0 & 3 & 0 & 1 & $\frac{9}{2}$ & 7 & 3394 & 11743 & 14100 & 219 & 637 & 970 & 41 & 61 & 135 & 1855 & 1726 & 1695 & 5 & 12 & 21 \\
\hline Sucre & 1 & 3 & 1 & 0 & 3 & 8 & $\frac{1264}{3403}$ & 6580 & 6746 & 82 & 453 & 521 & 29 & 47 & 170 & 512 & 693 & 629 & 2 & 12 & $\frac{12}{8}$ \\
\hline Tolima & 0 & $\frac{1}{10}$ & 0 & 0 & 4 & $\frac{3}{25}$ & 3403 & 11352 & 14890 & 54 & 290 & 294 & 21 & 49 & 87 & 1843 & 1547 & 1616 & 1 & 14 & 8 \\
\hline 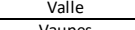 & 2 & 10 & 2 & 0 & 18 & 25 & 8533 & 39934 & 32822 & 112 & 728 & 728 & 21 & 67 & 77 & 4431 & 6028 & 4946 & 2 & 36 & 22 \\
\hline $\begin{array}{l}\text { auupes } \\
\text { Vichada }\end{array}$ & $\frac{1}{2}$ & $\frac{8}{0}$ & $\frac{1}{2}$ & $\frac{0}{0}$ & $\frac{0}{1}$ & 0 & $\frac{15}{21}$ & $\frac{93}{205}$ & $\frac{116}{159}$ & $\frac{2}{3}$ & $\frac{10}{6}$ & $\frac{10}{5}$ & $\frac{0}{0}$ & 0 & 0 & $\frac{15}{4}$ & $\frac{18}{31}$ & $\frac{15}{8}$ & $\frac{1}{0}$ & $\frac{1}{1}$ & $\frac{1}{1}$ \\
\hline Total nacional & 343 & 1673 & 343 & 7 & 208 & 241 & 94630 & 429907 & \begin{tabular}{l|l|}
454660 \\
\end{tabular} & 2980 & 14183 & 15051 & 325 & 1153 & 1559 & 48618 & 56526 & 52725 & 81 & 535 & 497 \\
\hline
\end{tabular}

Tema central

Situación Nal.

Mortalidad

Trazadores

Sarampión

Brotes

Tablas
2 instituto 


\begin{tabular}{|c|c|c|c|c|c|c|c|c|c|c|c|c|c|c|c|c|c|c|}
\hline \multirow[b]{2}{*}{ Departamento } & \multicolumn{3}{|c|}{$\begin{array}{l}\text { Mortalidad Perinatal y Neonatal } \\
\text { Tardia }\end{array}$} & \multicolumn{3}{|c|}{ Parálisis Flácida } & \multicolumn{3}{|c|}{ Parotiditis } & \multicolumn{3}{|c|}{ Sindrome de Rubeola Congénita } & \multicolumn{3}{|c|}{ Tos Ferina } & \multicolumn{3}{|c|}{ Varicela } \\
\hline & 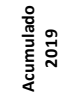 & 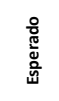 & 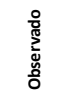 & 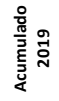 & 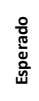 & 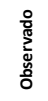 & 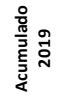 & 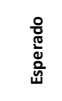 & 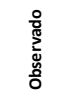 & 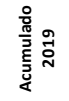 & 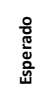 & $\begin{array}{l}\text { 윰 } \\
\text { 总 } \\
\text { 응 }\end{array}$ & 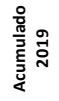 & 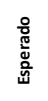 & 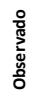 & 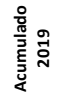 & $\begin{array}{l}\circ \\
\frac{0}{0} \\
\frac{0}{0} \\
\frac{8}{4}\end{array}$ & 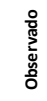 \\
\hline Amazonas & 0 & 1 & 1 & 0 & 0 & 0 & 0 & 1 & 8 & 0 & 0 & 0 & 0 & 3 & 0 & 0 & 9 & 5 \\
\hline Antioquia & 20 & 79 & 121 & 0 & 0 & 0 & 19 & 105 & 122 & 0 & 0 & 0 & 2 & 83 & 2 & 44 & 694 & 265 \\
\hline Arauca & 0 & 4 & 1 & 0 & 0 & 0 & 1 & 2 & 15 & 0 & 0 & 0 & 0 & 3 & 0 & 22 & 29 & 36 \\
\hline Atlantico & 7 & 29 & 33 & 0 & 0 & 0 & 0 & 15 & 7 & 0 & 0 & 0 & 0 & 1 & 0 & 0 & 123 & 114 \\
\hline Barranquilla & 4 & 32 & 32 & 0 & 0 & 0 & 0 & 22 & 13 & 0 & 0 & 0 & 0 & 2 & 0 & 16 & 213 & 92 \\
\hline Bogota & 113 & 252 & 304 & 0 & 0 & 0 & 61 & 295 & $\frac{13}{484}$ & 1 & 0 & 1 & 11 & $\frac{2}{129}$ & 11 & 52 & 1828 & $\frac{92}{416}$ \\
\hline Bolivar & 13 & 33 & 54 & 0 & 0 & 0 & 0 & $\frac{250}{6}$ & $\frac{404}{5}$ & 0 & 0 & $\frac{1}{0}$ & 0 & 4 & 0 & 8 & $\begin{array}{l}1020 \\
94\end{array}$ & 43 \\
\hline Boyaca & 6 & 22 & 21 & 1 & 0 & 1 & 2 & 27 & 17 & 0 & 0 & 0 & 0 & 7 & 0 & 6 & 171 & 48 \\
\hline Buenaventura & 2 & 4 & $\frac{2}{2}$ & 0 & 0 & 0 & 0 & 0 & 0 & 0 & 0 & 0 & 0 & 1 & 0 & 0 & 0 & 0 \\
\hline Caldas & 0 & $\frac{4}{9}$ & 8 & 0 & 0 & 0 & 3 & $\begin{array}{ll}7 \\
\end{array}$ & 9 & 0 & 0 & 0 & 0 & 17 & 0 & 6 & 107 & 38 \\
\hline Caqueta & 1 & 8 & 8 & 0 & 0 & 0 & 0 & 5 & 1 & 0 & 0 & 0 & 1 & 2 & 1 & 3 & 47 & 20 \\
\hline Cartagena & 12 & 52 & 44 & 0 & 0 & 0 & 2 & 11 & 9 & 0 & 0 & 0 & 0 & 5 & 0 & 15 & 57 & 67 \\
\hline Casanare & 3 & 9 & 4 & 0 & 0 & 0 & 1 & 2 & 1 & 0 & 0 & 0 & 0 & 2 & 0 & 2 & 38 & 14 \\
\hline Cauca & 16 & 36 & 55 & 0 & 0 & 0 & 3 & 14 & 9 & 0 & 0 & 0 & 0 & 5 & 0 & 0 & 93 & 19 \\
\hline Cesar & 3 & 24 & 30 & 1 & 0 & 1 & 0 & 14 & 5 & 0 & 0 & 0 & 0 & 5 & 0 & 7 & 53 & 43 \\
\hline Choco & 1 & 10 & 21 & 0 & 0 & 0 & 0 & $\frac{14}{0}$ & 0 & 0 & 0 & 0 & 0 & 3 & 0 & 0 & 4 & 8 \\
\hline Cordoba & 4 & 36 & 35 & 0 & 0 & 0 & 1 & 25 & 16 & 0 & 0 & 0 & 0 & 3 & 0 & 29 & 178 & 143 \\
\hline Cundinamarca & 27 & 62 & 109 & 1 & 0 & 1 & 9 & 42 & 68 & 0 & 0 & 0 & 3 & 17 & 3 & 33 & 503 & 155 \\
\hline Guainia & 0 & 1 & 0 & 0 & 0 & 0 & 0 & 0 & 0 & 0 & 0 & 0 & 0 & 1 & 0 & 0 & 1 & 0 \\
\hline Guajira & 3 & 33 & 46 & 0 & 0 & 0 & 0 & 4 & 0 & 0 & 0 & 0 & 0 & 1 & 0 & 10 & 39 & 53 \\
\hline Guaviare & 0 & 1 & 0 & 0 & 0 & 0 & 0 & 0 & 0 & 0 & 0 & 0 & 0 & 1 & 0 & 0 & 6 & 5 \\
\hline Huila & 14 & 46 & 40 & 0 & 0 & 0 & 4 & 13 & 42 & 0 & 0 & 0 & 0 & 13 & 0 & 5 & 119 & 38 \\
\hline $\begin{array}{l}\text { Magdalena } \\
\end{array}$ & 8 & 19 & 27 & 0 & 0 & 0 & 2 & 7 & 6 & 0 & 0 & 0 & 0 & 2 & 0 & 16 & 44 & 56 \\
\hline Meta & 6 & 18 & 15 & 0 & 0 & 0 & 0 & 12 & 9 & 0 & 0 & 0 & 0 & 4 & 0 & 11 & 129 & 50 \\
\hline Nariño & 10 & 28 & 52 & 0 & 0 & 0 & 2 & 9 & 16 & 0 & 0 & 0 & 2 & 11 & 2 & 10 & 187 & 72 \\
\hline Norte Santander & 5 & 17 & 22 & 0 & 0 & 0 & 8 & 27 & 57 & 0 & 0 & 0 & 4 & 15 & 4 & 14 & 224 & 90 \\
\hline $\begin{array}{l}\text { Putumayo } \\
\end{array}$ & 1 & 7 & 17 & 0 & 0 & 0 & 0 & 1 & 0 & 0 & 0 & 0 & 0 & 2 & 0 & 1 & 27 & 8 \\
\hline Quindio & 2 & 5 & 15 & 0 & 0 & 0 & 2 & 3 & 6 & 0 & 0 & 0 & 0 & 1 & 0 & 8 & 83 & 25 \\
\hline Risaralda & 2 & 15 & 7 & 0 & 0 & 0 & 1 & 6 & 5 & 0 & 0 & 0 & 0 & 4 & 0 & 3 & 93 & 46 \\
\hline San Andres & 0 & 1 & 0 & 0 & 0 & 0 & 0 & 2 & 1 & 0 & 0 & 0 & 0 & 1 & 0 & 7 & 14 & 28 \\
\hline Santa Marta D.E & 4 & 20 & 21 & 0 & 0 & 0 & 5 & 2 & 14 & 0 & 0 & 0 & 0 & 2 & 0 & 6 & 39 & 14 \\
\hline Santander & 9 & 26 & 31 & 0 & 0 & 0 & 1 & 19 & 7 & 0 & 0 & 0 & 1 & 11 & 1 & 7 & 291 & 69 \\
\hline Sucre & 4 & 18 & 26 & 0 & 0 & 0 & 0 & 20 & 6 & 0 & 0 & 0 & 1 & 15 & 1 & 27 & 135 & 90 \\
\hline $\begin{array}{l}\text { Tolima } \\
\end{array}$ & 9 & 23 & 31 & 0 & 0 & 0 & 3 & 12 & 13 & 0 & 0 & 0 & 0 & 7 & 0 & 10 & 165 & 56 \\
\hline Valle & 30 & 60 & 115 & 0 & 0 & 0 & 4 & 30 & 32 & 0 & 0 & 0 & 1 & 17 & 1 & 28 & 665 & 210 \\
\hline Vaupes & 0 & 0 & 0 & 0 & 0 & 0 & 0 & 0 & 0 & 0 & 0 & 0 & 0 & 1 & 0 & 0 & 2 & 0 \\
\hline Vichada & 0 & 2 & 2 & 0 & 0 & 0 & 0 & 0 & 0 & 0 & 0 & 0 & 0 & 1 & 0 & 0 & 3 & 1 \\
\hline $\begin{array}{l}\text { Total nacional } \\
\end{array}$ & 343 & 1044 & 1371 & 3 & 0 & 3 & 134 & 760 & 1003 & 1 & 0 & 1 & 26 & 402 & 26 & 406 & 6507 & 2437 \\
\hline
\end{tabular}

Tema central

Situación Nal.

Mortalidad

Trazadores

Sarampión

Brotes

Tablas 


\begin{tabular}{|c|c|c|c|c|c|c|c|c|c|c|c|c|c|c|c|c|c|c|c|c|c|c|}
\hline \multirow[b]{2}{*}{ Departamento } & \multicolumn{2}{|c|}{ Chagas agudo } & \multicolumn{2}{|c|}{ Difteria } & \multicolumn{2}{|c|}{ Leishmaniasis } & \multicolumn{2}{|c|}{ Leptospirosis } & \multicolumn{2}{|c|}{ Mortalidad Materna } & \multicolumn{2}{|c|}{$\begin{array}{c}\text { Mortalidad por IRA en } \\
\text { menores de } 5 \text { años }\end{array}$} & \multicolumn{2}{|c|}{ 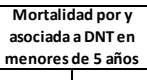 } & \multicolumn{2}{|c|}{ Sarampión/Rubeola } & \multicolumn{2}{|c|}{ Siffilis congénita } & \multicolumn{2}{|c|}{ Siffilis gestacional } & \multicolumn{2}{|c|}{ Tetanos accidental } \\
\hline & 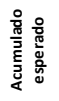 & 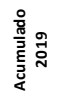 & 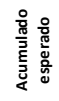 & 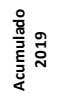 & 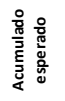 & 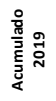 & 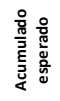 & 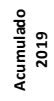 & 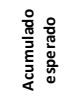 & 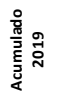 & 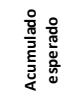 & 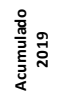 & 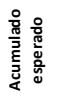 & 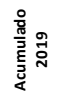 & 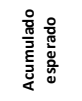 & 总 & 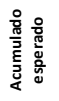 & 总 & 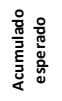 & 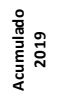 & 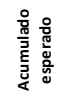 & 量 \\
\hline Amazonas & 0 & 0 & 0 & 0 & 1 & 0 & 0 & 0 & 0 & 0 & 1 & 0 & 0 & 0 & 0 & 0 & 1 & 0 & 0 & 0 & 0 & 0 \\
\hline Antioquia & 0 & 0 & 0 & 0 & 70 & 0 & 5 & 5 & 1 & 0 & 1 & 0 & 1 & 0 & 3 & 1 & 1 & 0 & 6 & 5 & 2 & 0 \\
\hline $\begin{array}{l}\text { Arauca } \\
\end{array}$ & 0 & 0 & 0 & 0 & 1 & 0 & 1 & 0 & 0 & 0 & 0 & 0 & 0 & 0 & 0 & 0 & 1 & 0 & 1 & 1 & 1 & 0 \\
\hline 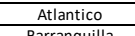 & 0 & 0 & 0 & 0 & 0 & 0 & 1 & 0 & 0 & 0 & 0 & 0 & 0 & 1 & 1 & 0 & 2 & 1 & 3 & $\frac{1}{2}$ & 1 & 0 \\
\hline Barranquilla & 0 & 0 & 1 & 0 & 0 & 0 & $\begin{array}{ll}1 \\
2\end{array}$ & 0 & 0 & 0 & 0 & 0 & 0 & 0 & 0 & 2 & 0 & 0 & 2 & 3 & 0 & 0 \\
\hline $\begin{array}{l}\text { Bogota } \\
\text { Bolyar }\end{array}$ & 0 & 0 & 1 & 0 & 0 & 0 & 2 & 0 & 1 & 0 & 1 & 0 & 1 & 0 & 3 & 10 & 3 & 0 & 13 & 6 & 0 & 0 \\
\hline Bolivar & 0 & 0 & 0 & 0 & 14 & 2 & 2 & 1 & 0 & 0 & 2 & 1 & 0 & 0 & 0 & 0 & 1 & 1 & 1 & 2 & 1 & 0 \\
\hline$\frac{\text { Boyaca }}{\text { Buenaventura }}$ & $\frac{0}{0}$ & 0 & $\begin{array}{l}0 \\
0\end{array}$ & 0 & 7 & 0 & 0 & 0 & $\frac{0}{0}$ & $\frac{0}{0}$ & 0 & $\frac{0}{0}$ & 0 & 0 & $\frac{0}{0}$ & $\frac{0}{0}$ & 0 & 0 & $\frac{2}{2}$ & 0 & 0 & 0 \\
\hline $\begin{array}{l}\text { Buenaventura } \\
\text { Caldas }\end{array}$ & $\frac{0}{0}$ & $\frac{0}{0}$ & $\begin{array}{l}0 \\
0\end{array}$ & $\frac{0}{0}$ & $\frac{3}{13}$ & $\frac{0}{0}$ & $\frac{2}{1}$ & $\frac{0}{0}$ & $\frac{0}{0}$ & $\frac{0}{0}$ & $\frac{0}{1}$ & 0 & $\frac{0}{0}$ & 0 & $\frac{0}{0}$ & $\frac{0}{0}$ & 0 & 0 & $\frac{2}{2}$ & $\frac{0}{1}$ & $\frac{0}{0}$ & $\frac{0}{0}$ \\
\hline Caqueta & 0 & 0 & 0 & 0 & 11 & 0 & 1 & 0 & 0 & 0 & 0 & 0 & 0 & 0 & 0 & 0 & $\frac{1}{0}$ & $\frac{1}{0}$ & 1 & 1 & 0 & 0 \\
\hline Cartagena & 0 & 0 & 0 & 0 & 0 & 0 & 2 & 0 & 0 & 1 & 1 & 1 & 0 & 0 & 0 & 5 & 1 & 1 & 2 & 1 & 1 & 0 \\
\hline Casanare & 0 & 0 & 0 & 0 & 0 & 0 & 0 & 0 & 0 & 0 & 0 & 0 & 0 & 0 & 0 & 0 & 2 & 0 & 2 & 0 & 0 & 0 \\
\hline Cauca & 0 & 0 & 0 & 0 & 5 & 0 & 1 & 0 & 0 & 0 & 1 & 0 & 0 & 0 & 1 & 0 & 1 & 1 & 1 & 1 & 1 & 0 \\
\hline $\begin{array}{l}\text { Cesar } \\
\text { Choco }\end{array}$ & $\frac{1}{0}$ & 0 & 0 & 0 & $\frac{1}{11}$ & $\frac{0}{1}$ & 1 & 0 & 0 & 0 & 1 & 0 & 1 & 0 & 0 & 1 & 2 & 0 & 3 & 1 & 1 & 0 \\
\hline $\begin{array}{l}\text { Choco } \\
\text { Cordobaa }\end{array}$ & $\frac{0}{0}$ & $\frac{0}{0}$ & $\frac{1}{0}$ & $\frac{0}{0}$ & $\frac{11}{15}$ & $\frac{1}{0}$ & $\frac{2}{0}$ & $\frac{0}{0}$ & $\frac{0}{0}$ & $\frac{0}{0}$ & $\frac{1}{2}$ & $\frac{0}{0}$ & $\frac{1}{1}$ & 0 & $\frac{0}{1}$ & $\frac{0}{0}$ & 2 & 0 & $\frac{1}{1}$ & $\frac{1}{1}$ & $\frac{0}{2}$ & $\frac{0}{0}$ \\
\hline Cundinamarca & 0 & 0 & 0 & 0 & 10 & 0 & 2 & 0 & 0 & 0 & $\frac{2}{1}$ & 0 & 0 & 0 & $\frac{1}{1}$ & 2 & $\frac{2}{0}$ & 0 & $\frac{1}{2}$ & $\frac{1}{2}$ & $\frac{2}{0}$ & 0 \\
\hline Guainia & 0 & 0 & 0 & 0 & 2 & 0 & 0 & 0 & 0 & 0 & 2 & 0 & 0 & 0 & 0 & 0 & 0 & 0 & 0 & 0 & 0 & 0 \\
\hline Guajira & 0 & 0 & 0 & 0 & 3 & 0 & 1 & 0 & 0 & 0 & 2 & 0 & 1 & 1 & 0 & 1 & 1 & 0 & 1 & 1 & 1 & 0 \\
\hline Guaviare & 0 & 0 & 0 & 0 & 24 & 0 & 2 & 0 & 0 & 0 & 0 & 0 & 0 & 0 & 0 & 0 & 1 & 0 & 0 & 0 & 0 & 0 \\
\hline Huila & 0 & 0 & 0 & 0 & 3 & 0 & 2 & 0 & 0 & 0 & 0 & 0 & 0 & 0 & 0 & 0 & 1 & 0 & 3 & 0 & 0 & 1 \\
\hline $\begin{array}{l}\text { Magdalena } \\
\text { Meta }\end{array}$ & 0 & 0 & 1 & 0 & $\frac{0}{30}$ & $\frac{0}{0}$ & 2 & 0 & $\frac{0}{0}$ & 0 & 0 & $\frac{2}{0}$ & $\frac{1}{1}$ & 0 & 0 & 0 & 2 & 0 & 3 & 0 & $\frac{1}{1}$ & 0 \\
\hline $\begin{array}{l}\text { Meta } \\
\text { Narīo }\end{array}$ & $\frac{0}{0}$ & $\frac{0}{0}$ & $\begin{array}{l}0 \\
0\end{array}$ & $\frac{0}{0}$ & $\frac{30}{24}$ & $\frac{0}{0}$ & $\frac{1}{0}$ & $\frac{0}{0}$ & $\frac{0}{0}$ & $\frac{0}{1}$ & $\frac{1}{0}$ & $\frac{0}{0}$ & $\frac{1}{0}$ & $\frac{1}{0}$ & $\frac{0}{1}$ & $\frac{1}{0}$ & $\frac{2}{1}$ & $\frac{0}{0}$ & $\frac{1}{4}$ & $\frac{0}{1}$ & $\frac{1}{1}$ & 0 \\
\hline Norte Santander & 0 & 0 & 1 & 0 & 21 & 0 & 0 & 0 & 0 & 0 & 1 & 0 & 0 & 0 & 1 & 2 & 1 & 1 & 2 & $\frac{1}{4}$ & 1 & 0 \\
\hline Putumayo & 0 & 0 & 0 & 0 & 10 & 0 & 0 & 0 & 0 & 0 & 0 & 0 & 1 & 0 & 0 & 0 & 0 & 0 & 1 & 1 & 1 & 0 \\
\hline Quindio & 0 & 0 & 1 & 0 & 0 & 0 & 1 & 3 & 0 & 0 & 0 & 0 & 0 & 0 & 0 & 0 & 1 & 1 & 2 & 0 & 1 & 0 \\
\hline $\begin{array}{l}\text { Risaralda } \\
\end{array}$ & 0 & 0 & 0 & 0 & 6 & 0 & 1 & 0 & 0 & 0 & 1 & 0 & 0 & 0 & 0 & 0 & 0 & 0 & 3 & 0 & 0 & 0 \\
\hline$\frac{\text { San Andres }}{\text { Santa Marta D.E. }}$ & $\frac{0}{0}$ & $\frac{0}{0}$ & $\frac{0}{0}$ & 0 & 0 & $\frac{0}{0}$ & 0 & $\frac{0}{0}$ & $\frac{0}{0}$ & $\frac{0}{0}$ & 0 & $\frac{0}{0}$ & $\frac{0}{0}$ & 0 & $\frac{0}{0}$ & 0 & 0 & 0 & 0 & 0 & $\frac{1}{0}$ & 0 \\
\hline $\begin{array}{l}\text { Santudvardav. } \\
\text { Santander }\end{array}$ & 0 & 0 & 0 & 0 & $\frac{1}{31}$ & 0 & 1 & 0 & 0 & 0 & 0 & 0 & 0 & 0 & 1 & 0 & $\frac{1}{1}$ & 0 & $\frac{1}{3}$ & $\frac{0}{2}$ & 1 & $\frac{0}{0}$ \\
\hline Sucre & 0 & 0 & 1 & 0 & 2 & 0 & 2 & 0 & 0 & 1 & 1 & 0 & 0 & 0 & 0 & 0 & 0 & 0 & 3 & 0 & 1 & 0 \\
\hline Tolima & 0 & 0 & 0 & 0 & 29 & 0 & 3 & 2 & 0 & 1 & 1 & 0 & 1 & 0 & 1 & 1 & 1 & 0 & 2 & 0 & 1 & 0 \\
\hline Valle & 0 & 0 & 0 & 0 & 2 & 0 & 9 & 1 & 0 & 1 & 1 & 1 & 2 & 0 & 2 & 0 & 3 & 0 & 6 & 4 & 0 & 0 \\
\hline Vaupes & 0 & 0 & 0 & 0 & 2 & 0 & 0 & 0 & 0 & 1 & 0 & 0 & 0 & 0 & 0 & 0 & 0 & 0 & 0 & 0 & 0 & 0 \\
\hline $\begin{array}{l}\text { Vichada } \\
\text { Total Nacional }\end{array}$ & $\frac{0}{1}$ & $\frac{0}{0}$ & $\frac{0}{1}$ & $\frac{0}{0}$ & $\begin{array}{c}5 \\
357 \\
\end{array}$ & $\frac{0}{3}$ & $\frac{0}{30}$ & $\frac{0}{12}$ & $\frac{0}{2}$ & $\frac{0}{6}$ & 1 & $\frac{0}{5}$ & 1 & $\frac{0}{3}$ & $\frac{0}{19}$ & $\frac{0}{26}$ & $\frac{0}{16}$ & $\frac{0}{7}$ & $\frac{0}{72}$ & $\frac{0}{41}$ & $\frac{0}{6}$ & $\frac{0}{1}$ \\
\hline
\end{tabular}

Tema central

Situación Nal.

Mortalidad

Trazadores

Sarampión

Brotes

Tablas

2 Saclodio 


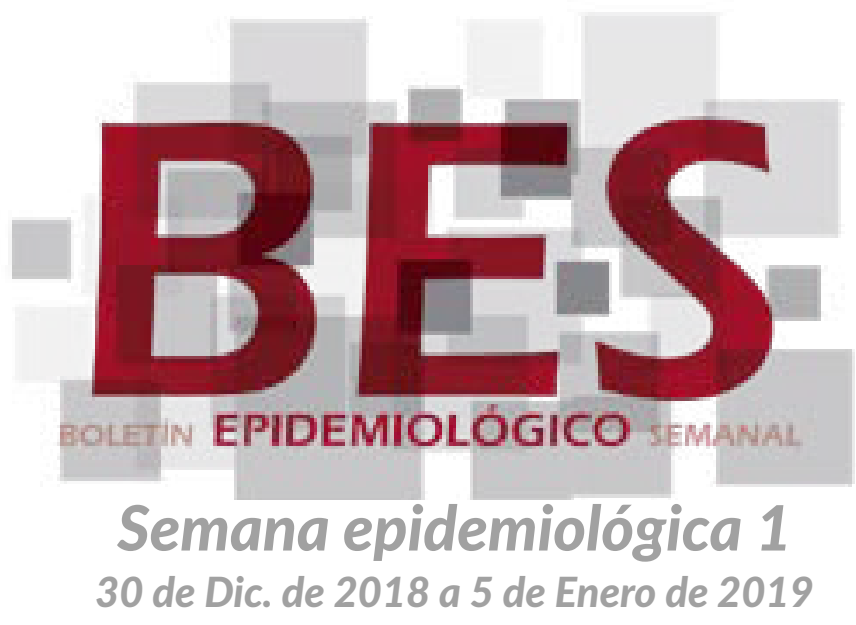

Expertos Temáticos

\begin{tabular}{|c|c|}
\hline & Expertos Temáticos \\
\hline $\begin{array}{r}\text { Dra. Martha Lucia Ospina Martínez } \\
\text { Directora General INS }\end{array}$ & $\begin{array}{l}\text { Situación Nacional } \\
\text { Gestor sistema de alerta temprana eri@ins.gov.co }\end{array}$ \\
\hline $\begin{array}{l}\text { Dr. Franklyn Edwin Prieto Alvarado } \\
\text { Director de Vigilancia y Análisis del Riesgo en Salud Püblica }\end{array}$ & $\begin{array}{l}\text { Mortalidad } \\
\text { Norma Lozanonnlozano@ins.gov.co }\end{array}$ \\
\hline $\begin{array}{l}\text { Dr. Oscar Pacheco García } \\
\text { Subdirector de Prevención Vigilancia y Control en Salud Pública } \\
\text { Dr. Hernán Quijada Bonilla }\end{array}$ & $\begin{array}{l}\text { Indicadores trazadores } \\
\text { Sara Gómez Romero sgomez@ins.gov.co }\end{array}$ \\
\hline $\begin{array}{l}\text { Dr. Hernán Quijada Bonilla } \\
\text { Subdirector de Análisis del Riesgo y Respuesta inmediata }\end{array}$ & $\begin{array}{l}\text { Orlando Castillo ocastilloins.gov.co } \\
\text { Brotes } \\
\text { Gestor sistema de alerta temprana eri@ins.gov.co }\end{array}$ \\
\hline $\begin{array}{r}\text { Una publicación del: } \\
\text { Instituto Nacional de Salud } \\
\text { de Vigilancia y Análisis del Riesgo en Salud Pública } \\
\text { Publicación en línea: ISSN 2357-6189 }\end{array}$ & $\begin{array}{l}\text { Tablas de mando } \\
\text { Verónica Tangarife vtangarife@ins.gov.co } \\
\text { Diana Rivera drivera@ins.gov.co }\end{array}$ \\
\hline 2018 & $\begin{array}{l}\text { Edición } \\
\text { Hernán Quijada Bonilla hquijada@ins.gov.co }\end{array}$ \\
\hline & $\begin{array}{l}\text { Diseño y diagramación } \\
\text { Alexander Casas acasasc@ins.gov.co }\end{array}$ \\
\hline
\end{tabular}

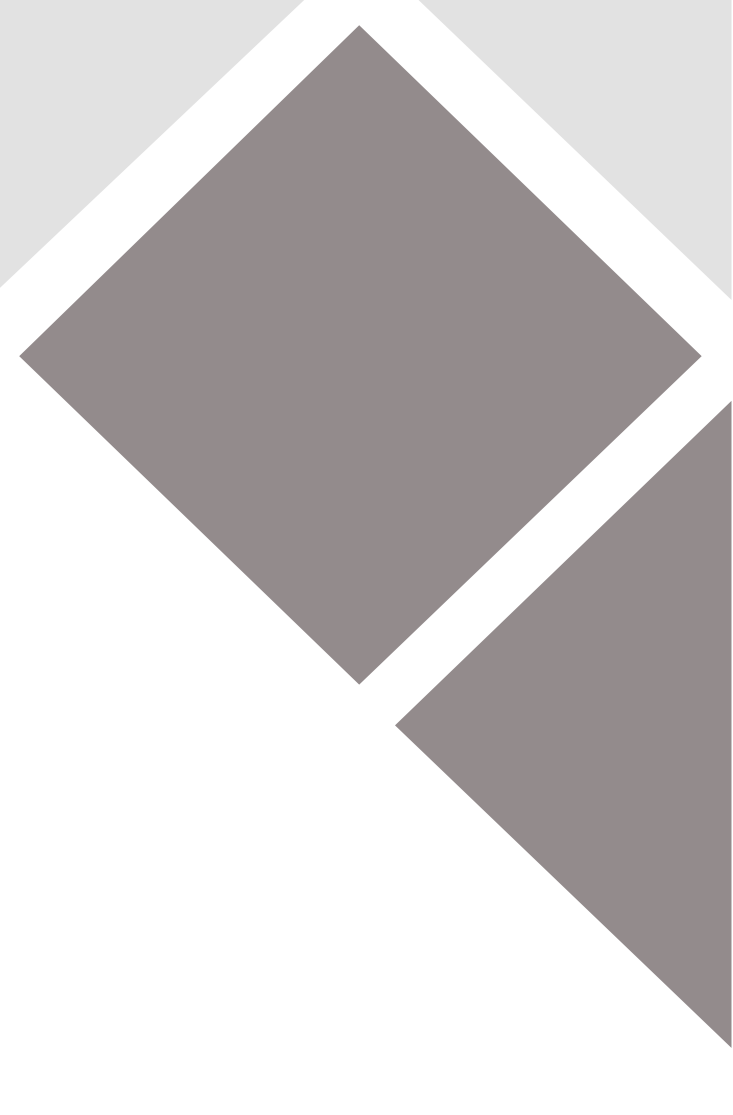

\section{GOBIERNO} DE COLOMBIA
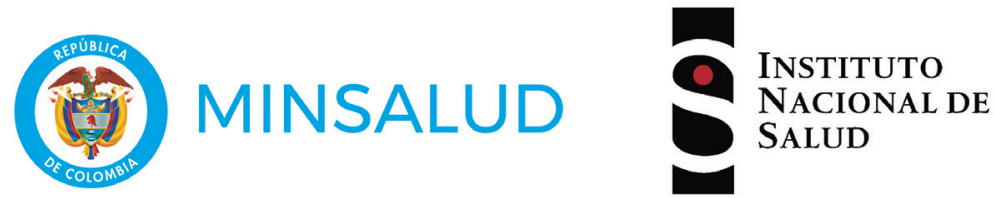

SIstema de vigIlancia en salud publıca (SIVIg|la). tI numero de casos, puede varıar despues de que se realızan unıdades de análisis en las ET para el ajuste y la clasificación de caso en cada evento. 